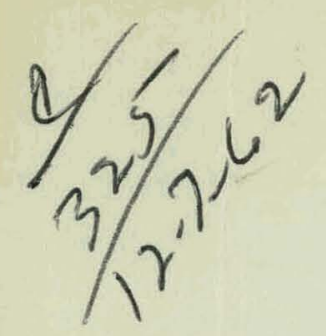

ORNL-3318

UC-32-Mathematics and Computers

TID-4500 (18th ed.)

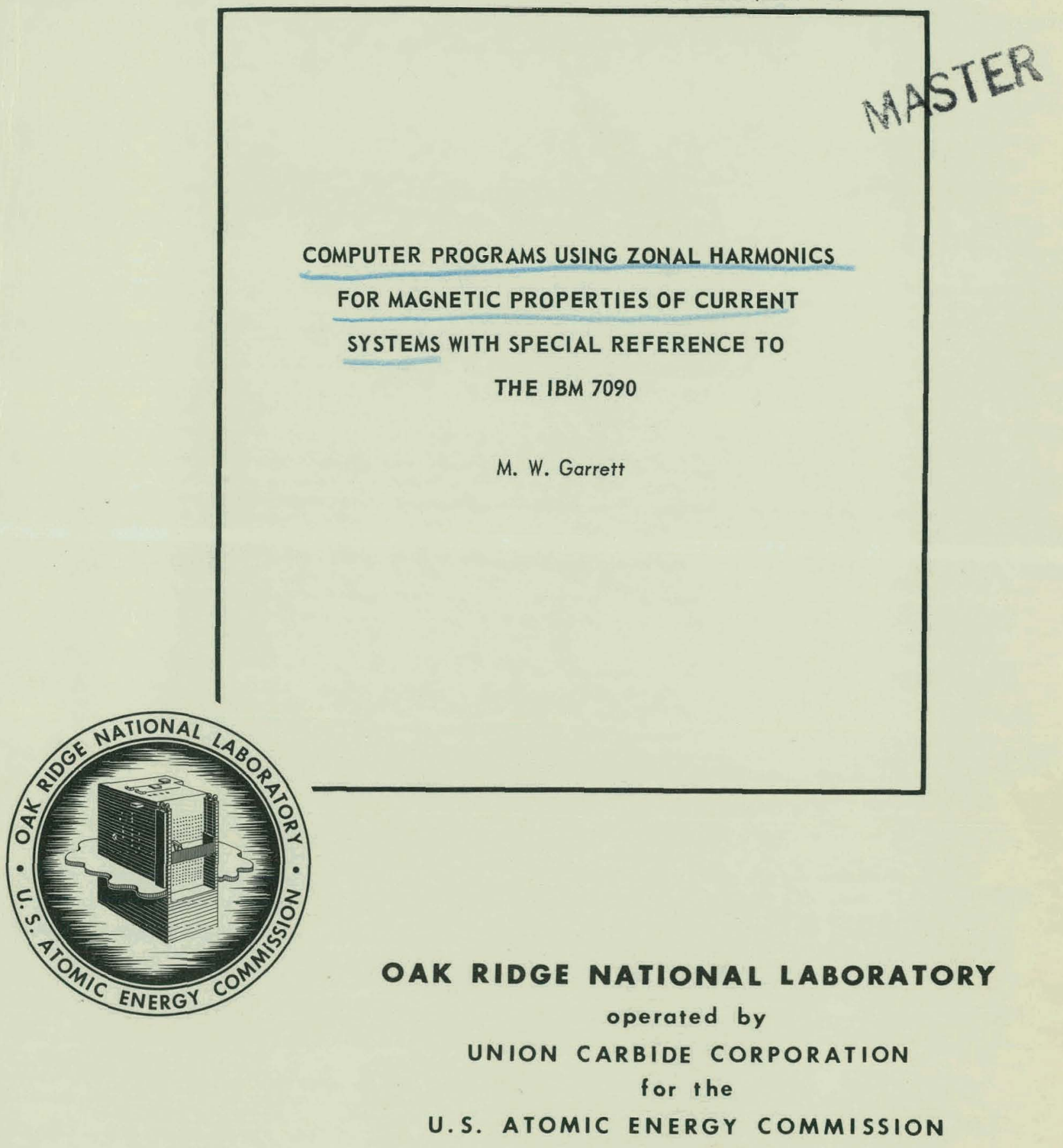




\section{DISCLAIMER}

This report was prepared as an account of work sponsored by an agency of the United States Government. Neither the United States Government nor any agency Thereof, nor any of their employees, makes any warranty, express or implied, or assumes any legal liability or responsibility for the accuracy, completeness, or usefulness of any information, apparatus, product, or process disclosed, or represents that its use would not infringe privately owned rights. Reference herein to any specific commercial product, process, or service by trade name, trademark, manufacturer, or otherwise does not necessarily constitute or imply its endorsement, recommendation, or favoring by the United States Government or any agency thereof. The views and opinions of authors expressed herein do not necessarily state or reflect those of the United States Government or any agency thereof. 


\section{DISCLAIMER}

Portions of this document may be illegible in electronic image products. Images are produced from the best available original document. 
Printed in USA. Price: $\$ 1.25$ Available from the

Office of Technical Services

U. S. Department of Commerce

Washington 25, D. C.

\section{LEGAL NOTICE}

This report was prepared as an account of Government sponsored work. Neither the United States, nor the Commission, nor any person acting on behalf of the Commission:

A. Makes any warranty or representation, expressed or implied, with respect to the accuracy, completeness, or usefulness of the information contained in this report, or that the use of any information, apparafus, method, or process disclosed in this report may not infringe privately owned rights; or

B. Assumes any liabilities with respect to the use of, or for damages resulting from the use of any information, apparatus, method, or process diselosed in this report.

As used in the above, "person acting on behalf of the Commission" includes any employee or contractor of the Commission, or employee of such contractor, to the extent that such employee or contractor of the Commission, or employee of such contractor prepares, disseminates, or provides access to, any information pursuant to his employment or contract with the Commission, or his employment with such contractor. 
Contract No. W-7405-eng- 26

THERMONUCLEAR DIVISION

COMPUTER PROGRAMS USING ZONAL HARMONICS FOR MAGNETIC PROPERTIES OF CURRENT SYSTEMS WITH SPECIAL REFERENCE TO THE IBM 7090

M. W. Garrett

DATE ISSUED

DEC $\quad 41962$

OAK RIDGE NATIONAL LABORATORY Oak Ridge, Tennessee

operoted by UNION CARBIDE CORPORATION

U. S. ATOMIC ENERGY COMMISSION 


\section{THIS PAGE}

\section{WAS INTENTIONALLY \\ LEFT BLANK}




\section{CONTENTS}

CHAPTER 1. THEORY.

The Traditional Microforms. Source and Field Harmonics ......................................................... 3

Restriction to Macroforms. Notation for Functions of Angle ...............................................................

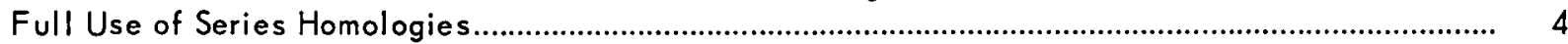

General Origin.

Two-Stage Analysis. Field and Source Equations ..............................................................................

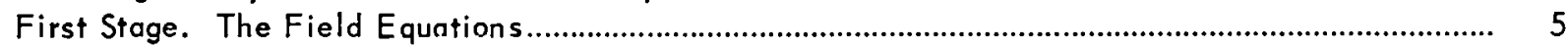

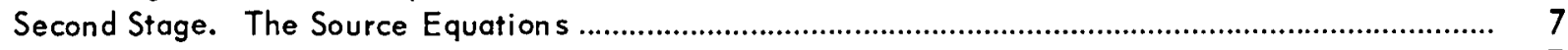

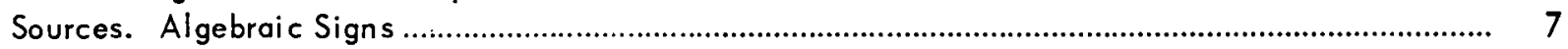

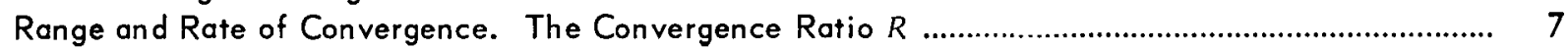

Effect of Polar Angle. Source Harmonics ... . ................................................................................. 9

Convergence in Practice. The Nomogram .................................................................................. 10

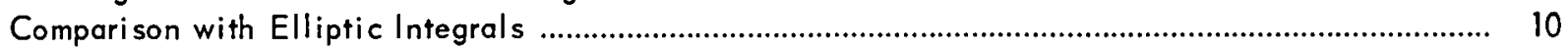

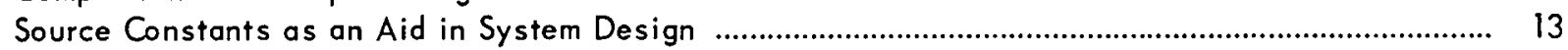

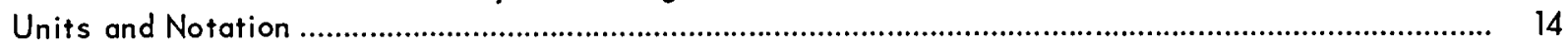

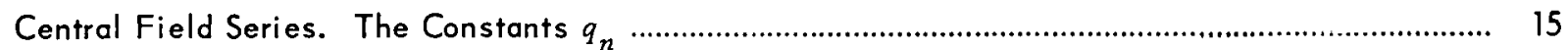

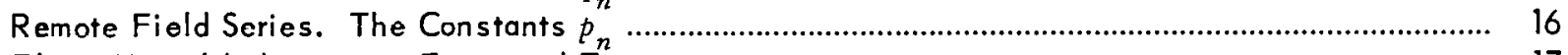

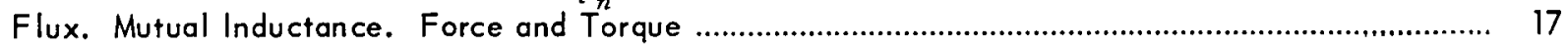

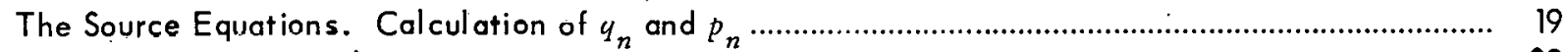

The Recurrence Formulas ......................................................................................................... 22

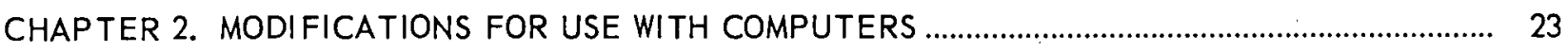

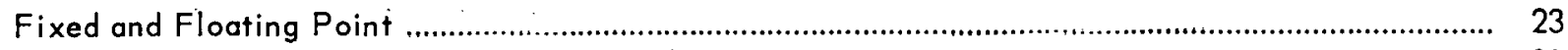

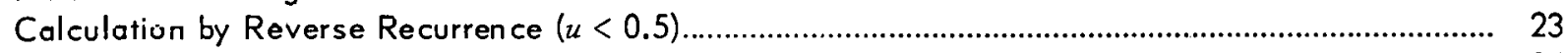

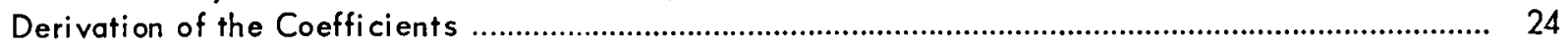

Limitation on Forward Recurrence............................................................................................. 24

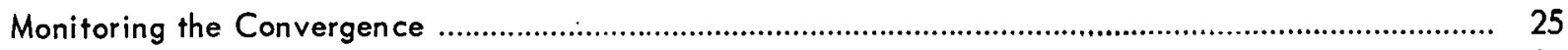

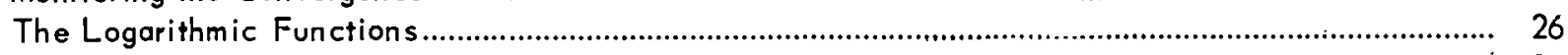

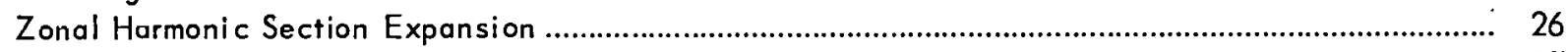

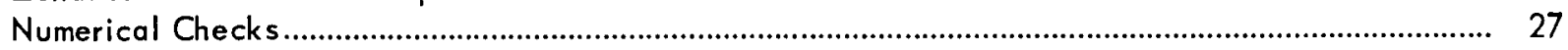

A Flexible Zonal Harmonic Computer Program ............................................................................... 28

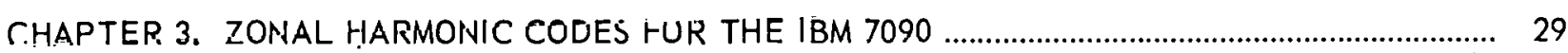

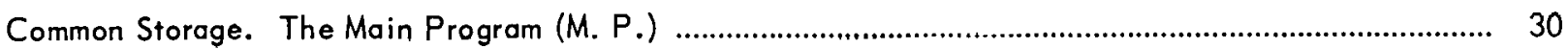

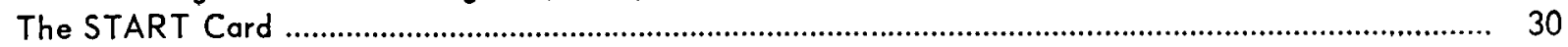

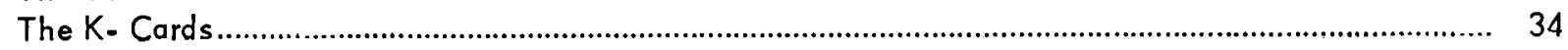

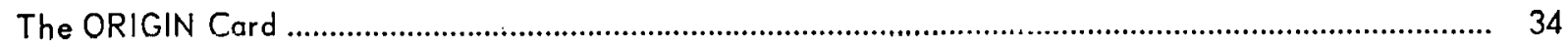

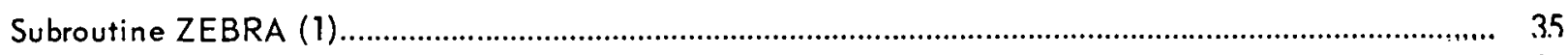

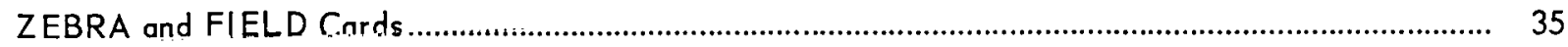

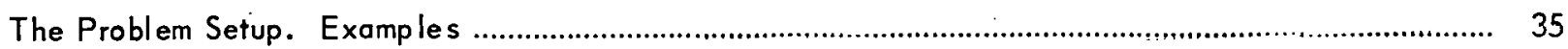

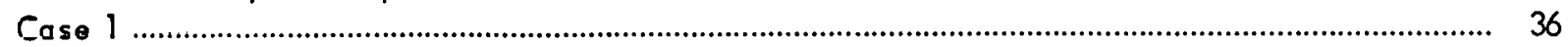

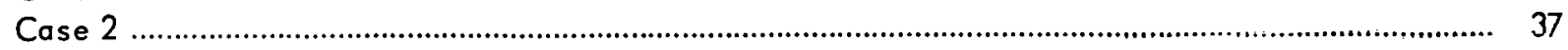

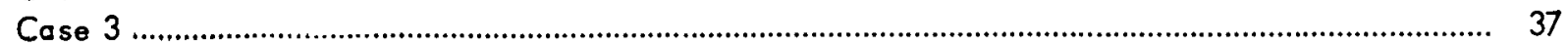




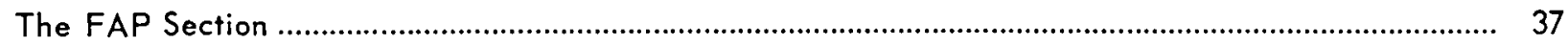

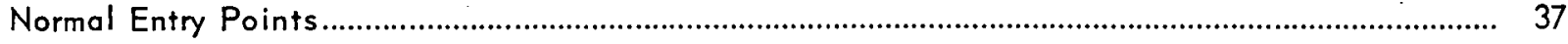

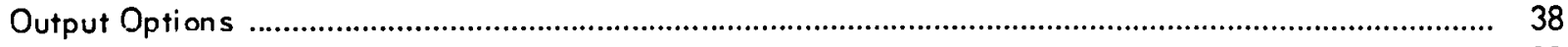

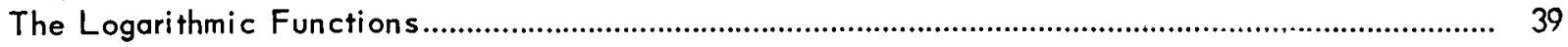

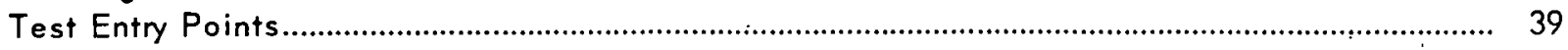

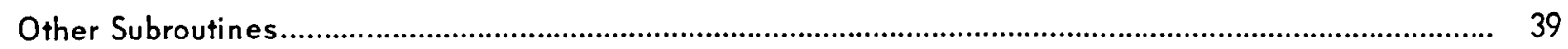

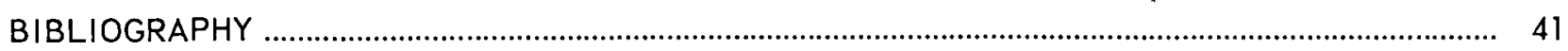




\title{
Computer Programs Using Zonal Harmonics for Magnetic Properties of Current Systems with Special Reference to the IBM 7090
}

\author{
M. W. Garrett ${ }^{\prime}$
}

\begin{abstract}
This report discusses the magnetic vector and scalar potentials, magnetic ficld components and the ir derivatives, and flux linkage for single current systems, and the mutual inductance, forces and torques between two such systems, whose axes are coplanar but not necessarily coincident. Each system may include a combination of coaxial loops, cylindrical or plane annular current sheets, and cylindrical coils of rectangular section.

Working equations are listed, following an outline of theory in a form that has considerable generality but is at the same time ideally suited to precise calculation. A discussion of errors and of means for reducing them leads to specific suggestions for use of the method with any programmed computer. An existing comprehensive program for the IBM 7090 is described in detail.

The rate of convergence of the harmonic series depends on the ratio $R$ of the polar radius of the field point to that of the current discontinuity nearest the origin. In some cases the precision reaches 1000 parts per million even at $R=0.95$,

improving to 1 or 2 parts at $R=0.80$. Since the origin is arbitrary, the practical range of the method often includes the whole space that is accessible to experiment. For very close approach to the windings one must resort to elliptic integral methods or their cquivalent.

Alternative programs that have been used are compared with the method of zonal harmonics, but they are slower by more than a factor of 10 when large numbers of field calculations must be made on a complex current system. One example is the problem of following individual ions through paths in the order of 100 turns in a field that is generated by 20 coaxial thick coils; available competing methods would have required too much computer time. The zonal harmonic method also has superior flexibility in attacking a wide range of problems. This is especially true for the calculation of rates of change, with respect to coordinates of the field or of the generating system, and for problems in which some property of a system must be not only calculated, but also adjusted to meet a specification.
\end{abstract}

\section{INTRODUCTION}

The magnetic properties of a current system, as considered here, include the magnetic scular and vector potentials and their derivatives, the magnetic flux lliruugh the system in an applied field, and the mutual inductance, forces, and torques bctween two such systems. The use of zonal harmonics restricts the discussion to axially symmetric systerns. A system may include

\footnotetext{
'Consultant, Swarthmore College.
}

any combination of coaxial circular current loops, cylindrical or plane annular current sheets, and cylindrical volume currents, which will usually be called loops or filaments, (thin) solenoids, disks or pancakes, and (thick) coils respectively, and will be referred to as elements of the system.

Chapter 1 of this report develops the theory in outline, with the working equations. The theory, equations and machine programs all depend on a two-stage analysis in which the field and the system that gives rise to it are separately treated. 
The coordinates of the field and of its sources never appear in the same equations or the same subroutines. The field equations and the source equations have in common only a set of "source constants" $q_{n}$ and $p_{n}$, whose definition depends on the concept of a "source." The constants may be defined either for a single source or for a complete system. This method is more general, more compact in formulation, and more efficient in practice than the conventional approach $(1,2)$. Since many of the concepts and equations are unfamiliar, as are the new source functions $U_{n}$ and $W_{n}$, Chapter 1 is a necessary prelude to the description of the computer codes.

Chapter 2 discusses modifications that are necessary or desirable when the equations are embodied in a machine program. Although this section contains some specific references to an existing zonal harmonic program and subroutines for the IBM 7090, most of the information should be useful to anyone who seeks to apply the same method in solving problems of the same or related types with other machines; or even with a desk calculator. ${ }^{2}$

Chapter 3 describes the 7090 codes in greater detail for those who may be able to use them, with or without changes. Many details in this section also should be helpful in organizing similar programs for other computers.

2 Most of the methods, special formulas, series coefficients and other aids presented in chap. 2 were developed in the course of the author's previous work on field design problems, using first the Burroughs E-101 and UDEC computers and later the UNIVAC, all with fixed point coding. This earlier work including the calculation of Table I (not previously published) was supported by the Army Research Office (Durham).

\section{APPRECIATION}

It is a pleasure to acknowledge my debt to a number of people in the course of this work: to Dr. W. F. Gauster, who suggested the project arid to whom I owe both encouragement and helpful criticism; to Carl Parker, who helped day by day in many woys, and who adapted the first version of the progrull lu lle culculation of ion orbite (5); th the staff of operaters and coordinators for whom the efficient mastery of the genie seemed always to be as much pleasure as duty; and to the half dozen or more patient mathematicions and computer experts who cheerfully dispensed first aid and comfort to a newcomer whenever the balance of victory wuvered belwein man and tho machine. 


\section{CHAPTER 1. THEORY}

\section{The Traditional Microforms. Source and Field Harmonics}

Limitations of space forbid the inclusion of full derivations. Little more can be done than to list the working equations. These however deviate sufficiently from traditional practice, in form, interpretation, and method of use that a reasonably full analysis of the underlying logic is indispensable. Most of the equations are deferred for convenience of reference to a point near the end of Chapter 1, while a summary of concepts, methods, and performance is first undertaken.

The method of expanding laplacian field series in zonal harmonics is old and well known. But since shortly after Maxwell's time a kind of underbrush has grown up in the zonal harmonic jungle that often obscures the trees. The criticism goes far beyond an objection to cumbersome and inefficient algebraic forms, and implies a widespread failure to notice correlations and to establish unifying principles. It has been a common practice to derive in isolation, and to compute from, a host of inflated special forms for special cases that will be referred to here as microforms. A general symbolic form such as $P_{4}^{\prime}(\theta)$ will be called a macroform. ${ }^{3}$ Its three most commonly used microform equivalents are $\frac{5}{2}\left(7 \cos ^{3} \theta-\right.$ $3 \cos \theta), \frac{5}{2} \cos \theta\left(4-7 \sin ^{2} \theta\right)$, and $5 / 2 r^{-3}\left(4 z^{3}-3 \rho^{2} z\right)$. The third form is properly a power series in the cotangent of the polar angle. To write out $P_{32}^{\prime}$ would require 16 terms, with many coefficients of about 15 digits. To compute it to two significant figures, seven or eight must be carried because of the mutual attrition of + and - terms.

The general term of a complete zonal harmonic series contains a product of two such harmonic functions, belonging to a source angle of the current system and a field point angle, respectively, together with powers of the two polar radii. The labor of computing from the microforms increases more rapidly than the square of the order, and most series are stopped even in derivations at the ninth or tenth order, that is at about the fifth term of the usual all-odd or all-even series.

Equally serious has been a failure to recognize the harmonic functions at all. Neglect of analogies that would surely have been discerned among the several sets of macroforms, and of homologies within the sets, accounts for many gaps and redundancies that have stood for half a century. The following sections will attempt to define under four heads the elements of a more systematic approach. ${ }^{4}$

\footnotetext{
${ }^{3}$ The names were suggested by the terms micro- and macro-instruction, which belong to the jargon of programming.

${ }^{4}$ Emphasis on these elements may be justified by a slightly overstated argument. Consider a set of magnetic quantities, $m$ in number, to include various field components, the vector potential, force, mutual inductance, etc. Let conventional microform series be separately derived for each of $n$ source types (loop, solenoid, disk, coil). Each harmonic factor of the general term, independently of the other, commonly occurs in any of three equivalent forms as noted above for $P_{4}^{*}$. Nearly always the origin is restricted in one of two ways, to remove terms of either odd or even order. This again doubles the number of possible microforms, which now stands at $18 m n$. If the solution of each case is carried out in two stages, with the source geometry and the field geometry segregated in separate equations written in macroform, expressions and calculations that are common to several cases are not repeated. There need be then only $m$ field equations and (counting both $p_{n}$ and $q_{n}$ ) $2 n$ source equations, to replace the entire set. The new equations are much shorter and far more lucid, while computing time is cut to a fraction through the use of recurrence routines.

The gaps that a more orderly system would fill are evident in any standard collection of field and mutual inductance microforms. But to trace correlations and identify the redundancies, or even to recognize the zonal harmonic exponsions for what they are, requires time ond some aid such as Table 1 of ref (1). For one example, see footnote 8 , hereafter.
} 


\section{Restriction to Macroforms. Notation for Functions of Angle}

For functions of the polar angle, apart from $s$ for sine and $u$ for cosine, the macroforms $P_{n}, P_{n}^{\prime}, U_{n}$, $w_{n}^{\prime}, P_{n}^{\star}, U_{n}^{*}$, and $W_{n}^{*}$ will be used exclusively. ${ }^{5}$ Though $P_{n}(u)$ is the Legendre polynomial in $u$ of order $n$, in most cases no argument will be written, and the defining Eq. (1) does not contain $u$. Each set of functions will be regarded as a set of magnitudes, characteristic of the polar angle, whose values after the first or second order may be found by successive iterations, and not as polynomials in the sine, cosine, or cotangent. Thus the angle itself will be written as argument, for example, $P_{n}(\theta)$, if it is not otherwise clear what angle is meant. The cosine $u$ appears in all the recurrence relations [Eqs. (4145)].

\section{Full Use of Series Homologies}

The microforms have usually been differentiated or integrated term by term, with respect to any coordinate, whereas such operations are performed on macroforms with the aid of general symbolic relations that may be derived from the series homology. (Note again that derivations cannot be included here.) The basic homology is concerned with $z$-differentiation. This can be seen most directly in the definition

$$
P_{n} \equiv \frac{(-1)^{n}}{n !} r^{n+1} \frac{\partial^{n}}{\partial z^{n}}\left(\frac{1}{r}\right)
$$

which uses the mixed cylindrical and polar coordinates $z, r$, but which actually defines $P_{n}$ as a function of the polar angle alone. Again, if $P_{n}^{\prime} \equiv \partial P /{ }_{n} \partial u$, the following relations can be proved:

$$
\left(1-u^{2}\right) P_{n}^{\prime}=\frac{(-1)^{n-1}}{(n-1) !} r^{n} \frac{\partial^{n} u}{\partial z^{n}}
$$

and

$$
\frac{\partial}{\partial z} r^{n} P_{n}=n r^{n-1} P_{n-1}, \quad \frac{\partial}{\partial \rho} r^{n} P_{n}=-r^{n-1} s P_{n-1}^{\prime} .
$$

For other useful homologies, see $(1,2)$. See also Eqs. (41-45), since the recurrence relations used for all calculations fall under this head. In effect, they reduce functions of all orders to 2-term polynomials.

${ }^{5} U_{n}$ and $W_{n}$ are new functions that were introduced in refs $(1,2)$ to deal with the case of thick coils. They permit the magnetic properties of such coils to be calculated as precisely, and almost as easily, as those of loops or solenoids. For the starred forms, see Eqs. (4). They are modified functions whose magnifudes never exceed unity, designed to replace $P_{n}, U_{n}$, and $W_{n}$, respectively, when iterations are carried out with a fixed decimal point。 


\section{General Origin}

All equations are referred to an arbitrary axial origin. This permits the field of a complete coaxial system to be coherently expressed by a single series, without adding up contributions from multiple origins. It also extends greatly the range of convergence of the central field series, since calculations may be referred to different origins in different parts of the field.

\section{Two-Stage Analysis. Field and Source Equations}

The efficiency of the zonal harmonic method depends on separating the variables. Since the functions $P_{n}, U_{n}$, etc. depend only on a polar angle, while all remaining variable factors of the harmonic terms are polar radii, this has already been done so far as the coordinates of each point are concerned. But there is a great gain in efficiency, both of formulation and of calculation, if the coordinates of field points and of sources (or in the cuse of mutual inductance, of primary and secondary sources) appear only in separate equations and are processed in separate subroutines of the machine program. Both the general theory and the practical problem are cleanly cut into two simpler parts, which are concerned with properties of the field and of the sources respectively (or of primary and secondary).

The separation permits a dramatic reduction in the number and complexity of the equations, a point that is elaborated in footnote 4. At the same time it clarifies the meaning since the elements that are essential to correlations and analogies appear explicitly in the equations.

\section{First Stage. The Field Equations}

Two types of field are first defined, the "central" and the "remote." Figure 1 shows a current system with two stepped coils numbered 1 and 2 . Let a spherical shell be drawn about an arbitrary axial origin, of the minimum thickness to include all parts of the current system between the spherical boundaries. The central ficld is then bounded by the inner sphere of radius $r_{\mathrm{min}}$ while the remote field extends trom the sphere of radius $r_{\text {max }}$ to infinity. Each type of field can be fully described by a set of field series, Eqs. (8) to (13) and (16) to (21), that contain no coordinates of the generating system. They assume only that the system has axial symmetry. Such general field equations are made possible by the severe restrictions imposed by Laplace's equation, the symmetry, and the spherical boundaries. They must of course contain a set of adjustable coefficients, pure numbers whose values must later be found from the system geometry, but except for these dimensionless magnitudes each field series is the same for a single loop as for the most complex coaxial system.

The adjustable constants, from the manner of their definition, might logically be called field constants. The constant of order $n$ for the central field is proportional to the axial derivative $\partial^{n} V_{0} / \partial z^{n}$ of the magnetic scalar potential at the origin. The remate constant of order $n$ is proportional to the strength of a fictitious ideal multipole of order $n$, at the origin, from which the $n$th term of the remote field series may be considered to arise. Individual constants of either set can be experimentally meas. ured without knowing the geometry of the current system, and in fact the theory does not exclude axially symmetric distributions of magnetized matter between the bounding spheres. 
UNCLASSIFIED

ORNL-LR-DWG 63014

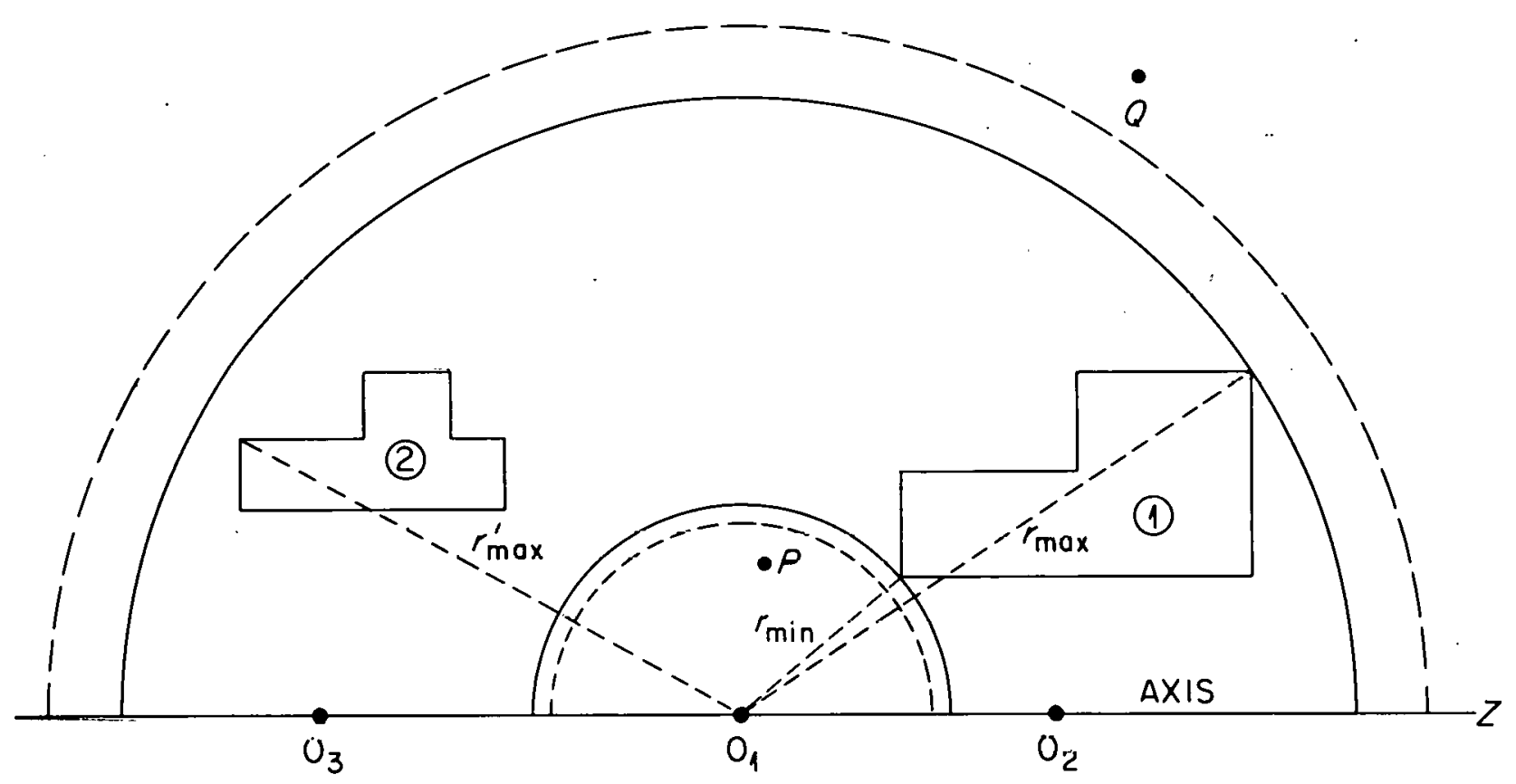

Fig. 1. Central and Remote Fields. Spheres of convergence.

In this work the constants are always computed from the geometry of the current system, and so are regarded as system constants. It will be seen in the next section that the contribution to any constant from each unitormly wound elemeñ (each loup, sulenuid, disk, or coil) of a cyctem is somputed frnm the coordinates of one, two, or four circles that mark the discontinuities in current density. Such circles are called "sources" of the field. Their contributions to the system constants of order $n$ are the source constants, $q_{n}$ for the central field and $p_{n}$ for the remote field. The system constants are dimenșionless scalar summations over all sources, that 15 , for uny yivell uldel $\dot{q}_{s}={\underset{i}{i}}_{q_{i} ;} p_{s}=\boldsymbol{\Sigma}_{i} p_{i}$. From this point on, $q_{n}$ or $p_{n}$, whether for a single source, a complete element, or an entire system, will usually be called a source constant of order $n$, suppressing the subscript $s$ or $i$ and dropping the term system constant. The $\boldsymbol{\Sigma}$ will not be written, but is to be understood implicitly in the field equations.

The flux through a system pluced in an arbitrary external field can be expressed in terms of the axial derivatives of the field and the constants $p_{n}$ of the system. The field need have no symmetry. From this expression, equations are derived for the mutual inductance, hence also forces and torques, between two axially symmetric systems, in terms of the q-constants of the primary and the $p$-constants of the secondary system. These also are field equations, since they contain no coordinates of sources. They cover in a few lines a wider range of cases than the entire standard repertoire of zonal harmonic microforms for force and mutual inductance. They fail in the presence of magnetized matter. 


\section{Second Stage. The Source Equations}

The second stage of analysis is to derive equations to calculate the source constants of any given system from its geometry. When computing, the order of the stages is reversed. A set of constants $q_{n}$ or $p_{n}$ is first calculated and stored, or in certain cases punched out on cards for later use. If the problem so requires, sets of constants are calculated. for each of several origins. Once computed, they are used without further need for the system geometry, to calculate all field components, the vector potential, forces, mutual inductances, etc.

The first step in deriving the constants is to expand the magnetic scalar potential of a single circular loop along the axis by Taylor's theorem, and to fit the result as a boundary condition to the corresponding central or remote zonal harmonic series. Thus $q_{n}$ and $p_{n}$ are found for the loop source. No further reference to any field equation is necessary. The superposition theorem for laplacian fields leads at once to simple expressions in closed form for the constants $q_{n}$ and $p_{n}$ of a cylindrical or annular current sheet and of a cylindrical volume current, through scalar integration of the expressions for $d q_{n}$ and $d p_{n}$ over the elementary current filaments, with respect to $z$ or to $\rho$ or both. The system source constants $q_{n}$ and $p_{n}$, as actually used in computing from the field equations, are then easily found by scalar summation over all the loops, solenoids and coils of the system.

\section{Sources. Algebroic Signs}

The definite integrals that express the $q$ - or $p$-constants for distributed currents contain no coordinates of internal current filaments, but only those of the current discontinuities, that is, the bounding eircular edges of the windings. Hence the concept of "sources," two in number for an ideal solenoid or disk, four for a cylindrical coil, that replace the physical distributed sources for all calculations. Except for thin loops carrying finite current, sources always occur in matched pairs of opposite sign but with the same strength, equal to the surface or volume current density. The source equations are for single sources of positive sign, bul the subscript $j$ of Fig. 2 is omitted.

Sign conventions for sources are shown in Fig. 2. For positive currents, sources at the right ends of solenoids and at the outer right corners of thick coils are positive. Signs in the latter case alternate round the section perimeter. The thick coil with stepped section requires only six sources when computing by hand. For the machine code, it is subdivided in either of the two ways shown. This adds a coincident redundant source pair at $S_{1}$ or at $S_{2}$, whose signs are shown inside the section.

\section{Range and Rate of Convergence. The Convergence Ratio $R$}

Several variables influence the details of convergence of the zonal harmonic series, but the terms of all serie's contain ascending powers of $R$, a ratio of two polar radii. In all cases, after a few terms the rate of convergence is dominated by the magnitude of $R$. The series fail in theory unless $R$ is less than one, and in practice it should rarely exceed 0.9 , occasionally perhaps 0.95 . For all series discussed in this paper, except for the double summations $(25,26)$, the combined secondary effect of all variables other than $R$ is to accelerate the smoothed rate in greater or lesser degree. 


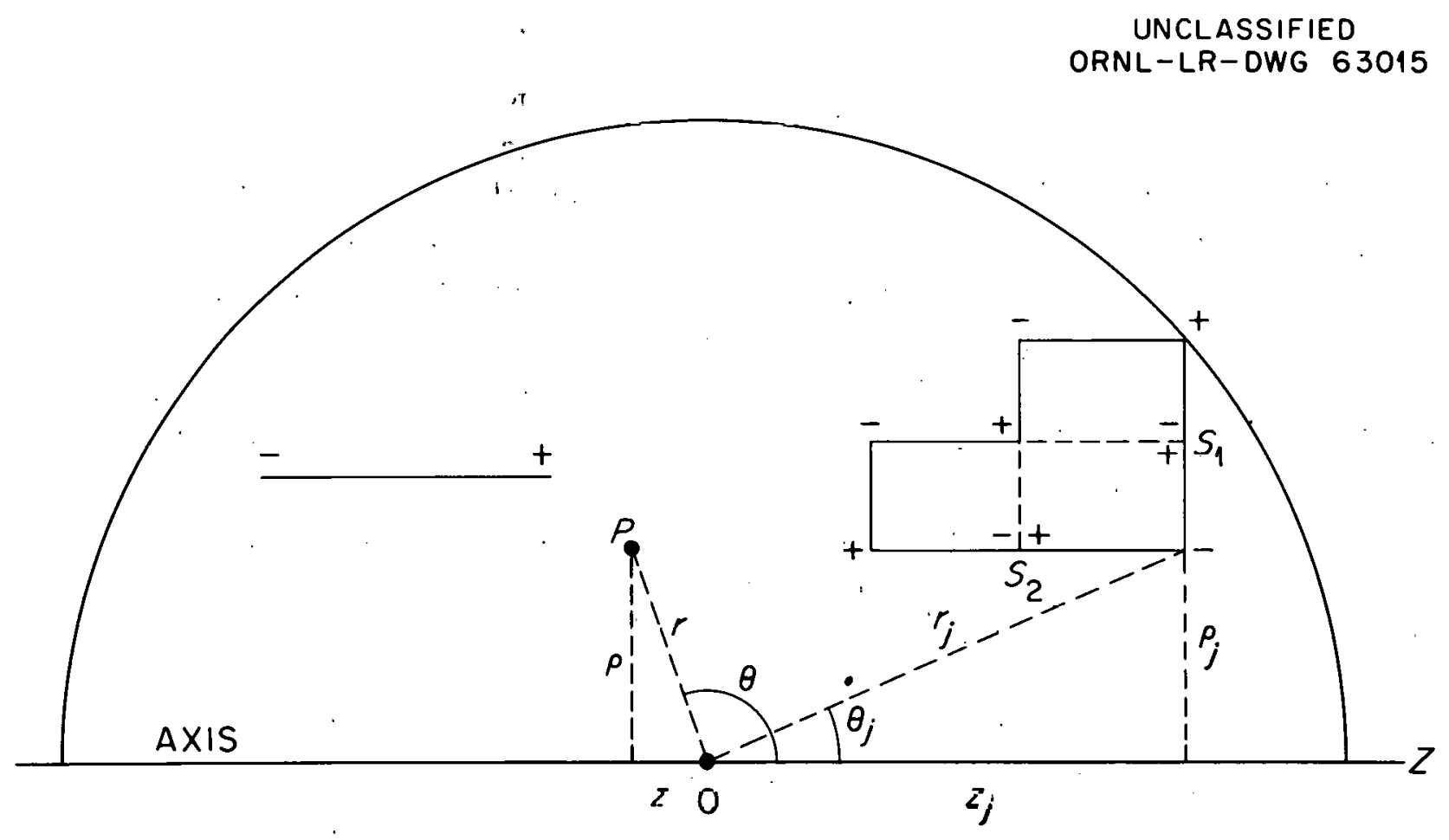

Fig. 2. Notation. Sign convention for sourcesa

Because of the separation of field and source equations, the powers of $R$ do not appear explicitly unless, for example, the values of $q_{n}$ from any of Eqs. (32) to (34) are substituted into one of the central

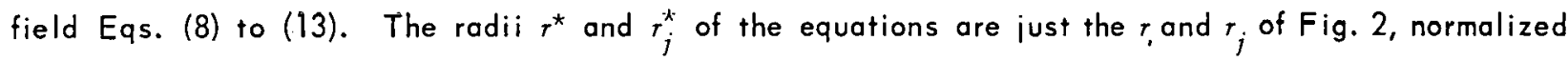
with respect to an arbitrary reterence length $r_{0}$. Evidently normalization does not affect the ratio $7 / r_{j}$. The $q$ 's for a complete system are summations over all sources. The source nearest the origin, whose radius in Figs. 1 and 3 is $r_{\text {min }}$ makes the dominant contribution to the $q$-summations of high order.

It follows that for the central field series, $R=r / r_{\mathrm{min}}$, where $r$ is the polar radius of the tield point. For the remote series, $R=r_{\max } / r$, where $r_{\max }$ may be seen in Figs. 1 and 3 . For mutual inductance, force or torque, $R=r_{\max }^{\prime} / r_{\mathrm{min}^{\prime}}$ where the prime belongs to the secondary and the radii are shown in Figs. 1 and 3. This means that the secondary must be wholly within the central field of the primary. $R$ can be varied over wide limits by moving the origin. Thus in Fig. 1, a series for the force or mutual inductance between coils 1 and 2 converges rapidly when the origin is placed at $\mathrm{O}_{2}$ or $\mathrm{O}_{3}$, but not if it remains at $O_{1}$. Again, if both coils constitute a single system, the total range of possible central fields can be greatly extended by computing from several origins in turn.. But to extend the remote field as far as possible, it is necessary to give up the single origin and to add two field components at each field point, computed separately for coils 1 and 2, from origins near $O_{2}$ and $O_{3}$ respectively. The same device may be used when computing force or mutual inductance. 


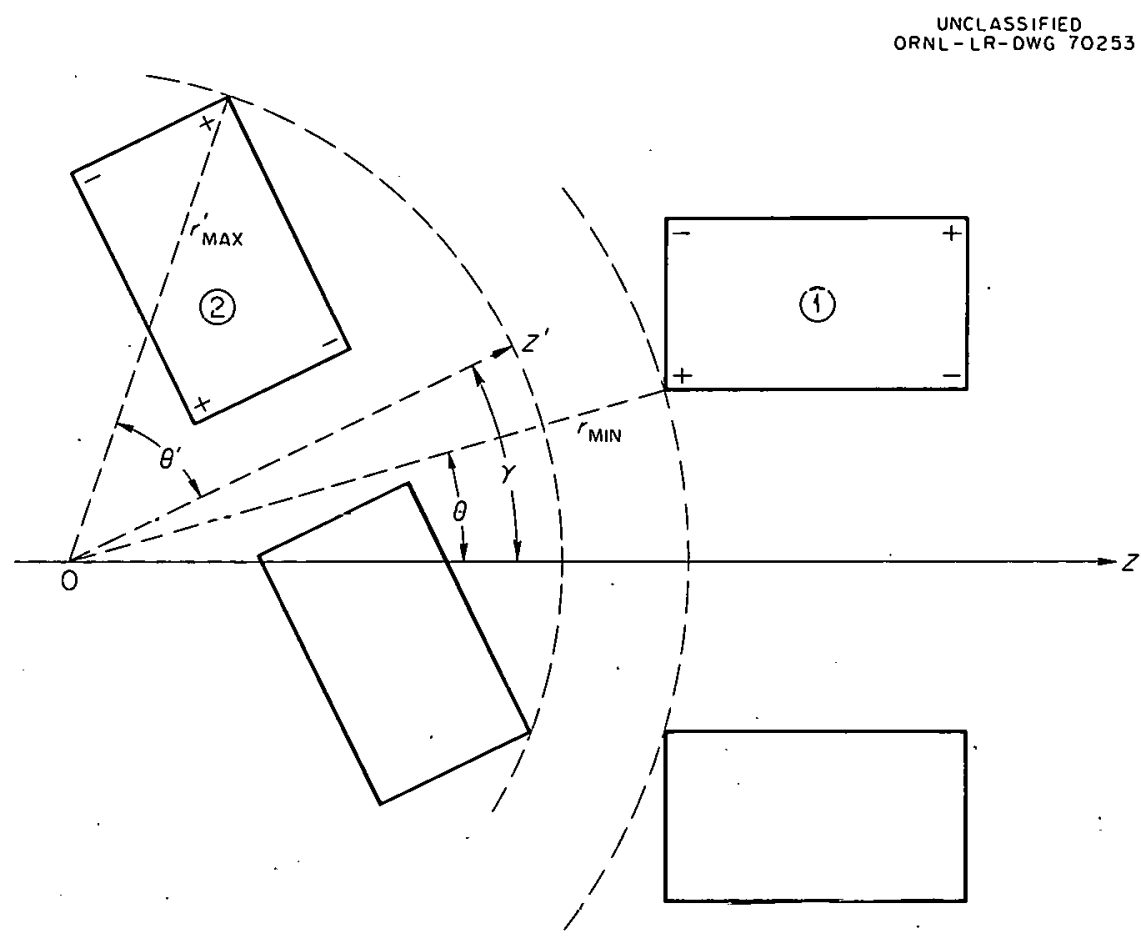

Fig. 3. Mutual Inductance Between Systems Whose Axes Intersect.

Note that convergence depends on the position of the sources and not of the physical currents. Consider a long solenoid of moderate wall thickness, with origin in the midplane. Its central field, bounded by a sphere through the inner source circles, includes much of the actual wound volume and of the space outside the coil. The colculations for $B_{\rho}$ and for vector potential require no correction in these regions. For $B_{2^{\prime}}$ it is only necessary to subtract $4 \pi j_{p} \times 10^{-7}$, where $j_{\rho}$ is the total linear current density $d i / d z$ between the axis and the field point, whose cylindrical coordinate is $\rho$.

\section{Effect of Polar Angle. Source Harmonics}

Term-by-term, as opposed to smoothed convergence, is strongly dependent on the polar angles of the dominant source and of the field point. The functions $P_{n}$ and $P_{n}^{\prime}$ occur in field equations, $P_{n}^{\prime}, U_{n}$, and $w_{n}$ (or their starred relatives) in source equations, all combined with powers of the polar radii. Though only $r^{n} P_{n}$ and $r^{-(n+1)} P_{n}$ are harmonics in the strict sense, all the functions of analognus form show similurities that make it convenient to refer to them loosely as harmonics. All the functions of polar angle are quasi-sinusoidal. They vary with angle and with order in a manner that parallels roughly the behavior of $\sin n \theta$ or of $\cos n \theta$, but with an amplitude that for large $n$ approaches $(n s)^{-1 / 2}$ for $P_{n}$ and $(n / s)^{1 / 2}$ for $P_{n}^{\prime}$. As $n$ increases, $U_{n+2}^{*}$ and $-W_{n-2}^{*}$ both approach $s P_{n}$ asymptotically.

Each term of a complete field series contains the product of a field harmonic and a source harmonic. The products of angular functions alone oscillate in sign in a variable sequence that depends on both 
polar angles, with possible extreme amplitudes that are either constant or diverge very slowly (nearly as $n$ ). The equations show also that most products contain one or more reciprocals of integers, which converge slowly (nearly as $n^{-1}$ ).

\section{Convergence in Practice. The Nomogram}

Because of the minor factors discussed above, the rate of convergence for a given value of $R$ varies slightly for different combinations of field and source equations (1). For example, source types listed in order of decreasing rate of convergence are: thick coils, solenoids or disks, and filaments. Field series for $B_{z}$ or $B_{\rho}$ converge somewhat faster than that for $\partial B_{\rho} / \partial z$, but more slowly than the vector potential series. Mutual inductance series converge faster than those for force, and these in turn faster than the $B_{z}$ or $B_{\rho}$ series, provided that the secondary origin is not displaced [as in Eqs. (25) and (26)].

The nomogram of Fig. 4 is designed to afford an estimate of the maximum truncation error to be expected for a range of values of $R=r / r_{\mathrm{min}}$, when a central field series for $B_{z}$ or $B_{p}$ is stopped at the variable order $n$, in the case of a coil with moderately thick section. The field of a thick coil arises from moderately diffuse currents, whose mean effective polar radius is appreciably greater than $r_{\mathrm{min}}$. Thin solenoids and a fortiori isolated loops are more concentrated sources, whose series converge somewhat more slowly. A rather loose chain of reasoning identities the error with $R^{n-2} U_{n}^{*} /(n-2)$, which can be read from the nomogram.

Powers of $R$ can be read directly from the right-hand scale, on a line through $R$ and the exponent $n-2$. For the field error, mark the point where this line cuts the index line, and read the fractional error an $n$ line with this mark and $U_{n}$.

The nomogram predicts maximum errors of 4,30 , and 180 parts per millıon, for $n-33$ unit $n=0.80$, 0.85 , and 0.90 . Errors observed in practice have been less than half of these figures. Axial forces between thick coils have been computed to within a few parts per million at $R=0.8,2$ or 3 parts in 10,000 at $R=0.90$, and better than $\frac{1}{2} \%$ at $R=0.95$. The results for mutual inductance are bettcr, about $0.1 \%$ at $R=0.95$.

The extension to 34 series terms, through the use of the recurrence formulas, has extended the range of the zonal harmonic series far beyond previously accepted limits. Chapter 2 will discuss several difficulties that were met in achieving this result with single precisiun coding: $\wedge$ rapidly inrreasing number of orders must be added for each small inercment in range from this point on, and it seems hardly worth the effort to compete with elliptic integral methods in the space that lies still closer to the windings.

\section{Comparison with Elliptic Integrals}

For precise calculation of fields and mutual inductances of loops and thin solenoids, with $R$ no greater than 0.4 or 0.5 , zonal harmonic series have long been used. They are ulliiest alwaye in mirmform and are frequently not recognized, and it is partly for this reason that the calculation by elliptic integrals is better known. When $R$ is greater than (say) 0.5 , a properly chosen series or iterative method 

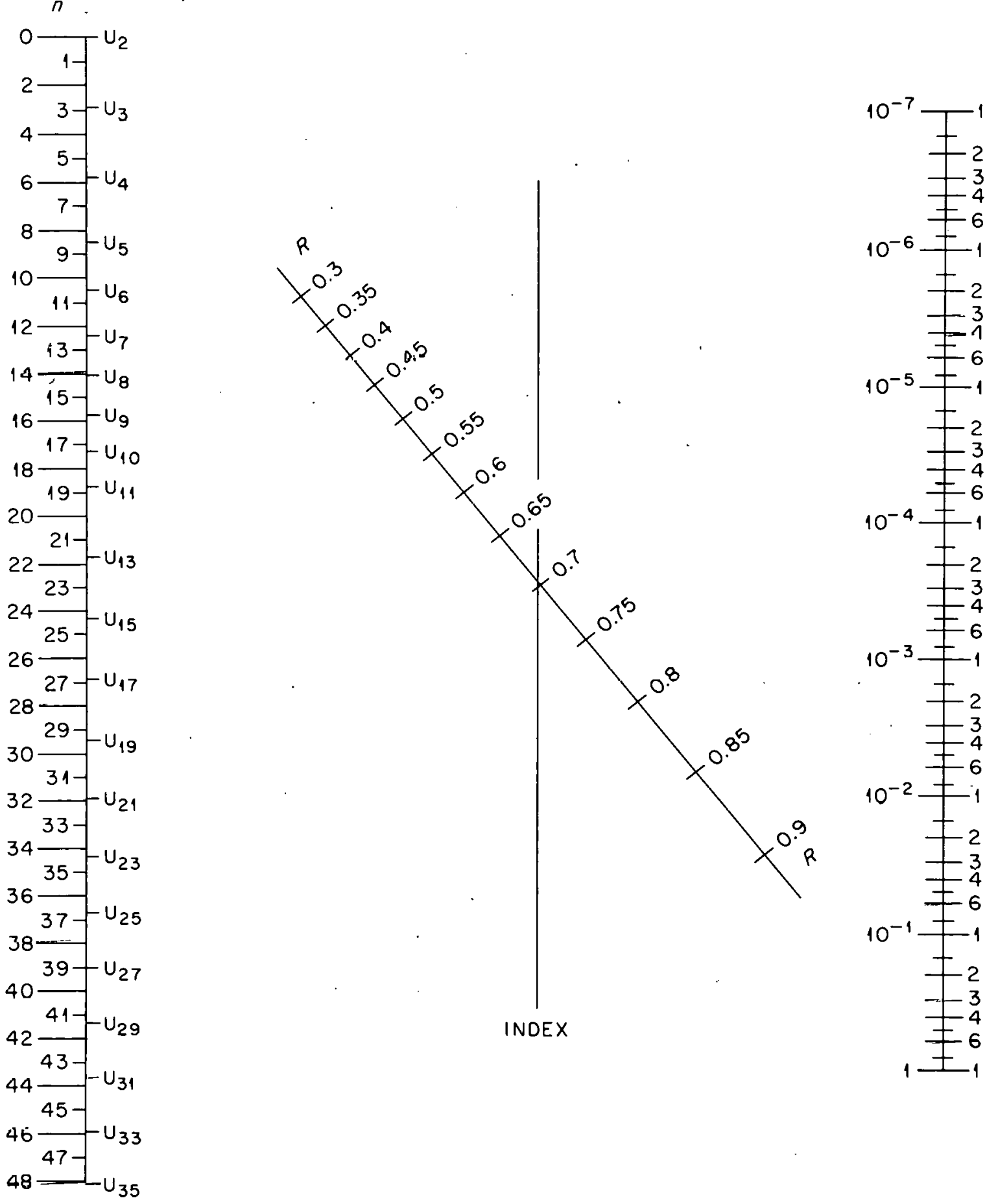

Fig. 4. To Find the Maximum Value of $\mathrm{U}_{n}^{\star} r^{n-2} /(n-2)$. 
using elliptic integrals is usually more economical for mutual inductance or for single field points. In fact, the limit on convergence is removed and fields may be computed at every point where the result has a valid physical meaning including points within the windings.

Nevertheless the zonal harmonic method has significant advantages. It reduces computing time in some cases by more than an order of magnitude. It is more versatile in solving a wide range of problems that depend on differentiation or integration with respect to field or source coordinates. Because it develops the field in continuity about an origin rather than by spot checks at a series of isolated points, it permits a direct approach to certain problems of field design. These advantages depend on the use of the series homologies, and on separation of the field and source geometries. They are further discussed in the following paragraphs.

Consider the "DCX-2" system, which generates a magnetic mirror field for thermonuclear experiments at the Oak Ridge National Laboratory. Fields in this system have been computed by several methods. The windings may be divided into 20 thick coaxial coils, which constitute 80 sources for the zonal harmonic method. If $n$ orders are retained, $80 n$ individual source constarits are computed by the recurrence formulas and summed into $n q$-constants, for each origin. For this highly elongated system, 10 to 12 origins are needed to compute the field components to one or two parts per million throughout some $70 \%$ of the total volime enclused by tho wintings, and to better than $1 / 10 \%$ over an additional $10 \%$, with $n=33$. The total number of constants to be computed and stored in the tirst stage of analysis is just under 400 . They may, if so desired, be punched out on cards for repeated use in field calculations as, for example, in orbit tracing.

All details of source geometry may now be discarded. To calculate $B_{z}, B_{\rho}, \Lambda$, or $\partial B_{\rho} / d z$ from $q_{n}$ of each tield polnt reyuites a fiold sories that is truncated on the average after fewer than $1 / 2 n$ terms, with a further economy in the case of the last three quantities since the $P_{n}^{*}$ functions of Eqs. (11-13) are computed only once fur all.

Fllintic integral methods are slower, in part because mnre sources are required, but principally because every detail of source geometry enters afresh into the field calculutions at ansh new point. The concept of an origin is irrelevant. The field at an off-axis point is built up as a sum of contributions from elementary circular current filaments, each of which depends on the cylindrical radii of the source and of the field point, and on their axial sepuruliuil. Tho coordinntes are not separable.

One formulation of the DCX-2 field, using elliptic integrals, replaced the 20 coils by 2154 loop sources, or about one per square inch of cross-section. At points near the windings, but still well within the range of the zonal harmonic code, the results were subject to small errors, presumably because the source nct was relatively coarse. Nevertheless the time required to compute a field net of several hundreds of points was estimated to be some 30 to 50 times greater than for the zonal hurmuric code. 6 An earlier version of the latter has been adapted by North and Parker (5) to trace ion orbits through as many as 100 turns in the DCX-2 field. This required some thousands of field calculations, and would scarcely have been practicable with the elliplic intcgral code.

\footnotetext{
${ }^{6} \mathrm{~T}$ ime estimates are approximate, since for these tests a flexible clock routine was not available.
} 
To reduce the number of sources per coil, it is desirable to integrate the elliptic integral loop equations with respect to the source coordinates. Integration with respect to $z$ can be accomplished in closed form. Thus two solenoid sources at the ends can replace a cylindrical sheet but the expressions for mutual inductance and for $B_{z}$ include an elliptic integral of the third kind. Integration with respect to $\rho$ has not been achieved, but the need can be circumvented by a Gaussian numerical quadrature. ${ }^{7}$ If an $n$-point formula is used, each thick coil is in effect replaced by $2 n$ solenoid sources. Though the method has greater speed and accuracy than one that assumes a set of uniformly distributed loops, it remains comparatively slow. With a system of $m$ coils, $2 m n$ sets of source coordinates still enter the field calculations at each point.

The flexibility of the zonal harmonic method in evaluating derivatives, with respect to linear and even to angular coordinates, is illustrated by the possibility of calculating mutual inductances when the secondary is tilted or when it is displaced, either axially or radially or both. Again, it required only six short lines of Fortran coding to add the calculation and readout of $\partial B{ }_{\rho} / \partial z$, when the code for $B_{\rho}$ and $A$ had been completed. The three functions are homologous. The gradient $\partial B_{z} / \partial z$ or any of several other derivatives presents no greater difficulty.

\section{Source Constants as an Aid in System Design}

A harmonic series gives a coherent description of the field as a continuum. Since it is a power series in the polar radius, a few nonvanishing terms of lowest order dominate the field throughout an extended region near the origin, and everywhere outside an enclosing sphere of moderate radius. Therefore the magnitudes of individual low-order source constants are immediately relevant to certain design problems. The number of orders that must be considered when the source constants are used as an aid to system design depends on the extent of the region of interest, more specifically on the convergence ratio $R$. As an aid to the use of the source constants for system design, the computer code includes an optional print of $\partial k_{n} / \partial z$ and $\partial k_{n} / \partial \rho$, where $k$ stands for either $q$ or $p$ and the rates of change are computed for displacements of each of the four boundaries of individual coils of the system.

In the following examples it will be assumed that all currents are symmetrical about the midplane, which restriets the suvice curislunls lu udd urders. There are also important cases of antisymmetry, the so-called cusp geometries, with a corresponding restriction to even orders. The uniformity of a central field increases with the number of successive constants $q_{3}, q_{5}, \ldots$, etc., that are suppressed.

\footnotetext{
7 This expedient was used to check the first pilot calculations made with the functions $U_{n}$ and $W_{n}$ against results obtained from the solenoid tunctions $\mu_{n}^{*}$ [ret $\left.(1), \mathrm{p} 1102\right]$. Downing has avoided the use of elliptic integrals altogether. He substitutes a Gaussian quadrature for the integration in azimuth around a circular loop, and combines it with an axial integration in closed form to find $B_{z}, B_{\rho}$, and $A$ for a solenoid source. More recently he has added a second Gaussian quadrature in radial depth, thus extending the use of his method to thick coils. The writer has completed, and expects to report on a program and subroutines that compute $B_{z}, B_{\rho}$, and $A$, together with mutual inductance and axial forces, for systems of loops, solenoids, and thick coils. This code uses a Gaussian quadrature of variable order in radiol depth, but relies otherwise on elliptic integrals throughout. Both programs discussed in this note can calculate fields at points within the winding space, and the second one can calculute the force and mutual inductance between two sections of a single coil that is divided by a transverse plane.
} 
Homogeneity to $1 / 10 \%$ out to $R=0.54$ on the axis and 0.63 in the midplane has been achieved by eliminating three orders [ref (1), p 1107]. Several hundred central field systems have been computed, using from two to four thick rectangular coils, to generate strong fields in which from one to three successive odd orders vanish.

A system for which $p_{3}, p_{5}, \ldots$, etc., are made to vanish generates a remote field that departs appreciably from that of an ideal dipole only at short distances. At the same time, the flux through the system in an arbitrary imposed field becomes more nearly proportional to the axial component of $B$ at the center of the system, ignoring field inhomogeneity. By suppressing three constants in a large search coil, used to report the value of $B$ at a point in a nonuniform field, sensitivity can be increased several hundredfold over that of a conventional small coil, for the same error. Mott has applied the concept of the multipole moments in a different way. He has simulated the remote field of DCX-2 by adjusting the currents and axial separations of two identical pairs of pre-existing coils to match the constants $p_{1}$ through $p_{7}(8)$.

Finally, let it be required to design the primary and secondary systems of a mutual inductor so that $M$ is accurately proportional to $\cos y$, where $\gamma$ is the angle of inclination of the axes. It is also desired that $M$ be nearly independent of moderate vector displacements of the secondary, which necessarily means that the force $F$ on the displaced secondary is negligible. The muin lerill of $M_{0}(y)$ is piupuliviral to $q_{1} p_{i} \cos \gamma$, with error terms proportional to $q_{3} p_{3}^{\prime}, q_{5} p_{5}^{\prime}, \ldots$, etc. The main error term of $M(r, 0)$ for a displacement $r_{1} \theta$ is proportional to $q_{3} p_{1}^{\prime} r^{2} P_{2}(\theta)$, and the analysis is readily extended to higher orders. [See Eqs. (23) to (26).] Since the constants $q_{n}$ of the primary and $p_{n}^{\prime}$ of the secondary are quite independent, while the products vanish with either factor, there is a considerable latitude for design even when several orders must be suppressed for both types of error.

\section{Units and Notation}

MKS units are used in all the equations of Chapter 1. The programs as written use the gauss and the centimeter for all the magnetic quantities, but with the option of describing the geometry in inches if so desired. These details are not relevant here, but are discussed in Chapter 3, under the heads of Scaling and Units and of Normalization (data card fields RX and BZ1).

For most equations the zonal polar coordinates $r, \theta$ are appropriate, though for input and output data, field components, and derivatives the cylindrical coordinates $z, \rho$ are more convenient (Fig. 2). The subscript $j$, which belongs to the $j$ th source, usually serves merely to identify a coordinate as that of a source rather than of a field point. Thus $\theta$ and $\theta_{j}$ often appear as the arguments of harmonic functions of the polar angles of field points and sources, respectively. The subscript may be dropped from any variable, and in the case of angles the argument also, whenever the omission can cause no confusion. In any case the notation $P_{n}(\theta), P_{n}^{+}(\theta)$, etc., never signifies a polynomial in $\theta$. The symbol $s$ is used for sine, and $u$ for cosine; $u$ may have either sign, but $s$ is necessarily positive.

Most lengths are scaled in terms of a suitably chosen unit or reference length $r_{0}$. Lengths so normalized are identified by an asterisk. It is usually convenient to make the starred source radii somewhat 
less than unity in central fields $\left(r_{0}<r_{\min }\right)$ and somewhat greater in remote fields $\left(r_{0}>r_{\text {max }}\right)$. The dominant $q$ - or $p$-constants are then slightly less than unity. The final results of the two-stage calculation are of course independent of the choice of $r_{0}$, but the intermediate magnitudes are sensitive to it.

An entirely distinct use of the asterisk is to distinguish three sets of functions of polar angles that appear in the field and source equations as they are listed below, replacing $P_{n}^{\prime}$ and the source functions $U_{n}$ and $W_{n}$ of $(1)$ and $(2)$ :

$$
\begin{aligned}
P_{n}^{\star} & \equiv s_{n}^{2} P_{n}^{\prime} / n, \\
U_{n}^{*} & \equiv U_{n} /(n-1), \\
W_{n}^{*} & \equiv W_{n} /(n+2) .
\end{aligned}
$$

The modified functions are preferred for the computer program, for reasons that are explained in Chapter 2. Their magnitudes never exceed unity.

The equations assume that the same current $i$ flows in each turn of the system. Thus the strength of an ideal circular current filament is $N i$, where the $N$ turns coincide and $N$ is not in general an integer. The strength of a thin solenoid source is the surface current density $N^{\prime} i$, where $N^{\prime}$ is the turn count per meter. The strength of a thick coil source is the volume current density $N$ " $i$, where $N$ "is the winding density in turns/meter ${ }^{2}$. Since the equations assume idealized uniform current sheets or volume currents, the dimensions of helical or thick cylindrical windings include the insulation of the outer turns. Every source must be given its proper sign, as previously defined and as shown in Fig. 2. For a more complete list of working equations that cannot be included in the following sections, and for derivations, (1) and (2) may be consulted.

\section{Central Field Series. The Constants $q_{n}$}

The central field is defined as a source-free axially symmetric laplacion field that is bounded by a sphere with center at the origin. Its scalar potential $V_{c}$ can always be expanded in the form

$$
V_{c}=\sum_{n=0}^{\infty} A_{n} r^{n} P_{n}(\theta)
$$

where the $A^{\prime}$ 's are constants that depend on the generating system and on the units employed. This expression must reduce on the axis to the Taylor series

$$
V_{a x}=\sum_{n=0}^{\infty} \frac{1}{n !} z^{n} \frac{\partial^{n} V_{0}}{\partial z^{n}},
$$

in which the zero subscript indicates that $V$ and its derivatives are evaluated at the origin, It is ronvenient to replace the $A$ 's of Eq. (5) by a set of dimensionless constants $q_{n}$, so defined that each is proportional to the axial derivative of corresponding order, with certain arbitrary factors:

$$
q_{n}=-\frac{r_{0}^{n} \times 10^{7}}{2 \pi i(n-1) !} \frac{\partial^{n} V_{0}}{\partial z^{n}} .
$$


On the axis, $r^{n} P_{n}(\theta)$ of Eq. (5) reduces to $z^{n}$. When the last three equations are combined, $V_{0}$ is set equal to 0 , and $r^{*}$ is written for the dimensionless ratio $r / r_{0}$, there results

$$
v_{c}=-2 \pi \times 10^{-7} i \sum_{n=1}^{\infty} \frac{1}{n} q_{n} r^{\star n} P_{n}(\theta) .
$$

The meaning and use of the constants $q_{n}$ and the reasons for calling them "source constants," have already been discussed. Equations (7) and (8) express the fact that an arbitrary central field is fully determined if all the axial derivatives $c^{n} V_{0} / \partial z^{n}$ are known at the origin. No direct reference to the source geometry is necessary.

From Eqs. (8) and (2),

$$
\frac{\partial^{m} V_{c}}{\partial z^{m}}=-2 \pi \times 10^{-7} i_{0}-m \sum_{n=1}^{\infty} \frac{(m+n-1) !}{n !} q_{m+n}{ }^{r^{\star n}} P_{n}(\theta) .
$$

This reduces when $m=1$ to

$$
-B_{z}=2 \pi \times 10^{-7} i r_{0}^{-1} \sum_{n=0}^{\infty} q_{n+1} r^{\star n} P_{n}(\theta)
$$

By setting $m=2$, it is easy to find the gradient $\partial B_{z} / \partial_{2}$.

From Eq. (8) and a known relation between the scalar and vector potentials,

$$
A=2 \pi \times 10^{-7} i \sum_{n=1}^{\infty} \frac{1}{n+1} q_{n} r^{\star n} s^{-1} P_{n}^{\star}(\theta) .
$$

The conventional notation for $s^{-1} p_{n}^{*}$ would be $(1 / 7 n)$ sill $\theta P_{n}^{\prime}[$ Eq. (4)]. Sucressive differentiations of (11) with respect to $z$ yield:

$$
\begin{gathered}
B_{\rho}=-2 \pi \times 10^{-7} i r_{0}^{-1} \sum_{n=1}^{\infty} \frac{n}{n+1} q_{n+1} r^{\star n} s^{-1} P_{n}^{\star}(\theta), \\
\frac{\partial B_{\rho}}{\partial s}=\frac{\partial B_{z}}{\partial \rho}=-2 \pi \times 10^{-7} i r_{0}^{-2} \sum_{n=1}^{\infty} n q_{n+2} r^{\star n} s^{-1} P_{n}^{\star}(\theta) .
\end{gathered}
$$

Equation (12) follows also from Eqs. (8) and (3). Series similar to those listed can be derived without difficulty for higher derivatives, and for derivatives with respect to polar coordinntes.

\section{Remote Field Series. The Constants $p_{n}$}

The remote field series are derived in analogous munner. The currently completed 7090 program computes for the remote field only the source constants $p_{n}$, but it would be easy to include field calculations by using the equations below. It is well known that the remote scalar potential can be expanded in the form

$$
V_{R}=\sum_{n=0}^{\infty} \mu_{n} r^{-(n+1)} P_{n}(\theta)
$$


where the $\mu$ 's are constants. They are often regarded as equivalent to a set of ideal multipoles of the origin; $\mu_{0}$ is a magnetic pole, $\mu_{1}$ a dipole, and so on. As before, it is convenient to replace the $\mu^{\prime}$ s by a set of dimensionless constants, arbitrarily defined. In this case,

$$
p_{n} \equiv(n+1) \mu_{n} / 2 \pi \times 10^{-7} i r_{0}^{n+1} \text {. }
$$

The $p_{0}$ is retained for generality. It appears for example in the first term of a mutual inductance series when, to accelerate convergence, the contributions from the two end planes of a single secondary solenoid or coil are referred to separate origins, each in its own plane. In passing, it should be noted that, when computing external fields close to the windings of a long system, best convergence is achieved by calculating the field of each coil separately, using a set of $p$-constants referred to an origin at the center of the coil. The zero order does not appear unless two origins are used for the field of a single coil, for example, one in each endplane.

The remote field equations, analogous to Eqs. (8-13) are:

$$
\begin{gathered}
V_{R}=2 \pi \times 10^{-7} i \sum_{n=0}^{\infty} \frac{p_{n}}{n+1} r^{\star-(n+1)} P_{n}(\theta) \\
\frac{\partial^{m} V_{R}}{\partial z^{m}}=(-1)^{m} 2 \pi \times 10^{-7} i r_{0}^{-m} \sum_{n=1}^{\infty} \frac{(m+n-1) !}{n !} P_{n-1} r^{\star-(m+n)} P_{m+n-1}(\theta) \\
B_{z}=2 \pi \times 10^{-7} i r_{0}^{-1} \sum_{n=1}^{\infty} P_{n-1} r^{\star-(n+1)} P_{n}(\theta) \\
A=2 \pi \times 10^{-7} i \sum_{n=1}^{\infty} \frac{p_{n}}{n+1} r^{\star-(n+1)} s^{-1} P_{n}^{\star}(\theta) \\
B_{\rho}=2 \pi \times 10^{-7} i r_{0}^{-1} \sum_{n=1}^{\infty} P_{n-1} r^{\star-(n+1)} s^{-1} P_{n}^{\star}(\theta) \\
\frac{\partial B}{\partial z}=\frac{\partial B_{z}}{\partial \rho}=-2 \pi \times 10^{-7} i r_{0}^{-2} \sum_{n=2}^{\infty} n P_{n-2} r^{*-(n+1)} S^{-1} P_{n}^{\star}(\theta)
\end{gathered}
$$

\section{Flux. Mutual Inductance. Force and Torque}

The flux of a central field through a coaxial circle is $2 \pi \rho A$, where $\rho$ is the radius of the circle, and $A$ can be found from Eq. (11). The flux that links a complete axially symmetric system can be calculated from the external coefficients $p_{n}$ of the system and the axial derivatives $\partial^{n} V_{0} / \partial z^{n}$, evaluated at the origin, of any external field in which it may be placed. All sources of the flux must be "remote" as previously defined, but the external field is otherwise arbitrary and may be devoid of symmetry. It is of course irrelevant whether the coil is energized. To derive Eq. (22), equate the energy of an arbitrary remote pole, at a point $r, \theta$ in the potential field (16) of the current system, to the energy of the system 
in the field of the pole. The derivatives of the pole field are found from (1). The equation reduces to

$$
\phi=-2 \pi \sum_{n=0}^{\infty} \frac{r_{0}^{n+1}}{(n+1) !} p_{n} \frac{\partial_{n} V_{0}}{\partial z^{n}}
$$

which implies that each p-constant responds, as it were by resonance, to a single field derivative. The principle has been applied to the design of large search coils which report the field, field gradient, etc., at a single point of an inhomogeneous field $(2,7)$.

The mutual inductance between two complete axially symmetric systems can be efficiently computed if the axes of the two systems are coincident or parallel, or, if they intersect in a common origin, as in Fig. 3. The mutual inductance between coaxial systems follows at once from Eq. (22), when the $p$ 's belong to the inner or secondary system, while the derivatives $\partial^{n} V_{0} / \partial z^{n}$ depend only on the $q^{\prime} s$ of the outer primary system, as given by Eq. (7). When the expression is generalized to include the case in which the axes intersect in the origin, at angle $y$, it becomes (3)

$$
M_{0}(\gamma)=4 \pi^{2} \times 10^{-7} r_{0}^{\prime} \sum_{n=0}^{\infty} \frac{p_{n}^{\prime} y_{n}}{n(n+1)} \bar{P}_{n}(\gamma) .
$$

The zero order term reduces to $p_{0}^{\prime} q_{0}$, since the $n$ in the denominator is to be dropped. This term is omitted except in the special case discussed in the next paragraph. The primed quantities belong to the secondary, and the unit for $M$ is the henry. If the axes coincide. omit $P_{n}(y)$. For the torque ahnut a transverse axis through the origin, change $P_{n}$ to $s P_{n}^{\prime}$, and multiply by $i i^{\prime}$. The equation assumes $r_{0}=r_{0}^{\prime} ;$ if in a special case the constants have been calculated with different reference radii for primary and secondary, insert the factor $\left(r_{0}^{*} / r_{0}\right)^{n}$ before $P_{n}$.

Equation (23) assumes that an origin can be found such that the secondary lies wholly within the central field of the primary, as in Fig. 3. Sometimes this requirement can be partially relaxed by adding together components of $M$ computed from two or more origins, usyally placed in the end planes of the secondary. For each origin, only the secondary sources in its own plane are then required to lie in the central field of the primary. The zero order must be retained when, and only when, multiple origins are used, and the summation must include every combination of a primary and a secondary source. Again, it is always permissible to interchange the complete set of $z$-coordinates of any primary element (loop, solenoid, or coil) with those of a secondary element, when computing the mutual inductance between the two. This device often extends the range of convergence.

When the values of $q_{n}$ and $p_{n}$ for loop sources are substituted from Eq. (32) and Eq. (37) into (23), the latter reduces to

$$
M_{0}(\gamma)=4 \pi^{2} \times 10^{-7} r^{\cdot} \sum_{n=1}^{\infty} \frac{n}{n+1}\left(\frac{r^{\prime}}{r}\right)^{n} P_{n}^{\star}(\theta) P_{n}^{\star}\left(\theta^{\prime}\right) P_{n}(\gamma) .
$$


This with minor changes in notation is Maxwell's formula for the mutual inductance between two loops whose axes intersect (6). ${ }^{8}$ Equation (23) is a versatile generalization of Maxwell's formula, from which, in fact, it can be derived by a superposition principle based on the concept of source constants. For the mutual inductance between two complete systems must be calculable as a scalar summation over all combinations of the elementary circular filament pairs. The angle $\gamma$ is common to all such pairs, while each product $p_{n}^{\prime} q_{n}$ of the source constants for the complete systems is just the scalar sum of all elementary products $d p_{n} d q_{n}$.

When the secondary system is arbitrarily displaced without rotation, the mutual inductance $M$ and the axial force $F_{z}$ on the secondary are laplacian scalar functions of the coordinates $r, \theta$ of the displaced secondary origin. It is therefore possible to find expressions for $M(r, \theta)$ and for its derivatives by reasoning that parallels the derivation of Eqs. (8) through (13). Since only the case of parallel axes will be considered here, $M_{0}$ is given by Eq. (23) without $P_{n}(\gamma)$. On the right side, $q_{n}$ alone is a function of $r$ and $\theta$. Substitute $M$ for $V$ in Eqs. (5) and (6), but omit Eq. (7) and evaluate the derivatives of $q_{n}$ with the aid of Eq. (28). The result is

$$
M(r, \theta)=4 \pi^{2} \times 10^{-7} r_{0} \sum_{n=0}^{\infty} \sum_{m=0}^{\infty} \frac{(n+m-1) !}{(n+1) ! m !} p_{n}^{\prime} q_{n+m}{ }^{\star m} P_{m}(\theta) .
$$

This equation reduces to (23) when $m=0$ or $r=0$, and for moderate displacements, it suffices to retain the first few orders only of $m$. Drop the zero order of $n$ unless multiple origins are used.

Forces and torques depend on the derivatives of $M$, with a factor $i i^{\prime}$. The axial and radial forces are related to $M$ as $B_{z}$ and $B_{\rho}$ of Eqs. (10) and (12) are to $V$ of Eq. (8):

$$
F_{z}(r, \theta)=4 \pi^{2} i i^{\prime} \times 10^{-7} \sum_{n=0}^{\infty} \sum_{m=0}^{\infty} \frac{(n+m) !}{(n+1) ! m !} p_{n}^{\prime} q_{n+m+1} r^{\star m} P_{m}(\theta) .
$$

For $F_{\rho}(r, \theta), m>0$, and $P_{m}(\theta)$ must be changed to $[-m /(m+1)]_{s}-1 P_{m}^{*}$ or $[-1 /(m+1)]_{s} P_{m}^{\prime}$. When the primary and secondary origins coincide $F_{\rho}=0$ and the axial force reduces to

$$
F_{z 0}=4 \pi^{2} i i^{\prime} \times 10^{-7} \sum_{n=0}^{\infty} \frac{1}{n+1} p_{n}^{\prime} q_{n+1} .
$$

The unit of force in these equations is the newton. As before, the zero order of $n$ is omitted unless multiple origins are used.

The Source Equations. Calculation of $q_{n}$ and $p_{n}$

In this section all equations for source constants apply to single positive sources, but the subscript $j$ appears only on the coordinates, to avoid possible confusion with field coordinates. The source

\footnotetext{
${ }^{0}$ The redundancy and lack of correlation that were discussed in footnote 4 may be illustrated by the microforms for special cases of Eq. (23) to be found in any standard collection of working formulas. Counting only the combination of two thin solenoids and of loop and solenoid there are nearly a dozen, including formulas considered to be distinct and attributed to Lorenz, Gray, Searle and Airey, Rosa, Roiti, Grover, Clem, Dwight, Snow, and others. Except for Snow's formula, all have sets of polynomials in the sine or cotangent that do not seem to have been recognized as harmonics, and even the exception has been similarly expanded for computing.
} 
constants of the field equations are summations, with due regard to algebraic sign, over all sources. The zero order $q_{0}$ is rarely used. It appears only in mutual inductance and force formulas, and then only when multiple origins are used, as explained in the last section.

Two general differential relations may be derived from Eqs. (7) and (15) respectively. They hold for an elementary current loop or for a complete axially symmetric system, and they have been used in the computer code to calculate rates of change of the source constants when a single source plane or a complete coil is displaced along the axis:

$$
\begin{gathered}
\partial q_{n} / \partial z=-n r_{0}^{-1} q_{n+1}, \\
\partial p_{n} / \partial z=(n+1)_{0}^{-1} p_{n-1} .
\end{gathered}
$$

Two further relations are physically obvious since a small axial increment to a thin solenoid is just a filament, while a radial increment to a thick coil is a thin solenoid. If $F, S$, and $C$ identify the source as filament, thin solenoid, or thick coil respectively, while $k_{n}$ is used in place of $q_{n}$ or $p_{n}$ since the equations are equally valid for either set,

$$
\begin{aligned}
& N \partial k_{n}(S) / \partial z=N^{\prime} r_{0} k_{n}(F), \\
& N^{\prime} \partial k_{n}(C) / \partial \rho=N^{\prime \prime} r_{0} k_{n}(S) .
\end{aligned}
$$

Equation (31) is used to calculate the effect on the source constants of displacing a cylindrical coil boundary rädiälly.

The elementary $q$-constants are those of a filamentary circular current element $N i$ at $r, \theta$, whose magnetic potential on the axis in the central field is $2 \pi \times 10^{-1} N i\left(1-u_{j}\right)$. The axial derivatives of this potential can be found from Eq. (3), and when they are substituted into Eq. (7) the result is

$$
q_{0}(F)=-N u_{j} ; \quad(n>0), \quad q_{n}(F)=N n r_{j}^{\star}-{ }^{n} P_{n}^{*}\left(\theta_{j}\right) .
$$

This basic result can be integrated with respect to $z$ to find $q_{n}(\Delta)$ for a thin solenoid. Introduction of the limits of integration suggests at once that the idealized helix can be replaced by a pair of fictitious equal sources, each of strength $N^{\prime} i$ but of opposite sign, coinciding with the circular edges of the equivalent current sheet.

$$
\begin{gathered}
q_{0}(S)=-N^{\prime} r_{0} r_{j}^{*}, \quad q_{1}(S)=N^{\prime} r_{0} u_{j}, \\
(n>0), \quad q_{n+1}(S)=-N^{\prime} r_{0} r_{j}^{*}-n P_{n}^{*}\left(\theta_{j}\right) .
\end{gathered}
$$


When Eqs. (33) are integrated in radial depth, that is with respect to $\rho$, the result for a thick coil is four equivalent sources, each of strength $N^{\prime \prime} i$, with signs that alternate round the perimeter, beginning with + at the outer right corner: ${ }^{9}$

$$
\begin{aligned}
q_{0}(C) & =-\frac{1}{2} N^{\prime \prime} r_{0}^{2} r_{j}^{\star 2}\left[s_{j}+u_{j}^{2} f\left(\theta_{j}\right)\right], \\
q_{1}(C) & =N^{\prime \prime} r_{0}^{2} r_{j}^{\star} u_{j} f\left(\theta_{j}\right), \\
q_{2}(C) & =N^{\prime \prime} r_{0}^{2}\left[s_{j}-f\left(\theta_{j}\right)\right], \\
f\left(\theta_{j}\right) & =\ln \left(\frac{1+s_{j}}{u_{j}}\right)=\ln \left(\rho_{j}+r_{j}\right)-\ln z_{j}=\sinh ^{-1}\left(\frac{\rho_{j}}{z_{j}}\right) . \\
(n>2), \quad q_{n}(C) & =\frac{N^{\prime \prime} r_{0}^{2}}{n-2} r_{j}^{\star}-(n-2) U_{n}^{\star}\left(\theta_{j}\right) .
\end{aligned}
$$

For hand computation, the function $f\left(\theta_{j}\right)$ can be read from a table of $\sinh ^{-1} x$. When computing it from the second logarithmic form, omit $\ln z_{j}$, which vanishes in summation for each end plane.

To derive Eqs. (33) without an integration, substitute Eq. (28) into Eq. (30), to yield

$$
n N_{F} q_{n+1}(S)=-N_{s}^{\prime} r_{0} q_{n}(F)
$$

and then replace $q_{n}(F)$ by the right side of Eq. (32). This method of arriving at Eqs. (33) suggests that the $q$-constants of a plane annular or disk winding $(D)$ can be found at once by treating it as a thin slice of a thick coil $(C)$ :

$$
N_{C}^{\prime \prime} r_{0} q_{n}(D)=-n N_{D}^{\prime} q_{n+1}(C)
$$

With the aid of this equation the source functions $U_{n}^{*}$ can be used to calculate the $q$-constants of disk coils (2).

The external source constants $p_{n}$ can be developed by closely similar reasoning. Only the results will be given here. All formulas include the zero order.

$$
\begin{gathered}
p_{n}(F)=N_{n r_{j}^{\star n+1}} P_{n}^{\star}\left(\theta_{j}\right) . \\
p_{n-1}(S)=\frac{N^{\prime} r_{0}{ }^{n}}{n+1} r_{j}^{\star{ }^{n+1}} P_{n}^{\star}\left(\theta_{j}\right),
\end{gathered}
$$

${ }^{9}$ The functions $U_{n}$ first appeared in ref (1), and $W_{n}$ in ref (2). [An error occurs in Eq. (54) of ref (1); everything following $u^{-1}$ should be enclosed in brackets.] For tables of these functions see footnote 10 . The modified functions $U_{n}^{*}$ and $W_{n}^{*}$ of Eq. (4), as used here, are calculated from Eqs. (44) and (45). See also Eq. (46). 


$$
\begin{gathered}
p_{n}(C)=\frac{N^{\prime \prime} r_{0}^{2}}{n+3} r_{j}^{\star n+3} W_{n}^{\star}\left(\theta_{j}\right), \\
N_{C}^{\prime \prime} r_{0} p_{n}(D)=(n+1) N_{D}^{\prime} p_{n-1}(C) .
\end{gathered}
$$

Much time can be saved in manual calculations if it is possible to place the origin in a plane of symmetry or antisymmetry of the coil system, or alternatively in the plane of the field point, thereby eliminating either odd or even terms of the complete harmonic series. Many types of problems, especially design problems, can be solved completely in this way. The 7090 program as written provides this economy automatically when it is possible, in the sections that compute $q_{n}$ and $p_{n}$. But this refinement was omitted from the program for the field equations, which was written after experience had shown the extreme speed of the machine.

\section{The Recurrence Formulas}

Four recurrence formulas for the harmonic functions of the angular coordinate of a source or of a field point are used in this work [Eq. (42) is given for reference only].

$$
\begin{aligned}
& r_{0}=1, \quad P_{1}=u, \\
& (n+1) P_{n+1}=(2 n+1) u P_{n}-n P_{n-1} \\
& P_{0}^{\prime}=0, \quad P_{j}^{\prime}=1 \text {, } \\
& n P_{n+1}^{\prime}=(2 n+1) u P_{n}^{\prime}-(n+1) P_{n-i}^{\prime} \text {. } \\
& P_{0}^{\star}=0, \quad P_{1}^{\star}=s^{2} \text {, } \\
& \Gamma_{n+1}^{*}=u P_{n}^{*}+\left(\frac{1}{n}-1\right) P_{n-1}^{*}+\left(1-\frac{1}{n+1}\right) u P_{n}^{\star}, \\
& U_{2}^{\star}=1, \quad U_{n+1}^{\star}=\left(1-\frac{1}{n}\right)\left(U_{n}^{\star}-s P_{n-1}^{\star}\right) u^{-1} \text { ， } \\
& W_{0}^{*}=1 / 2 s^{3}, \quad W_{n+1}^{*}=\left(1-\frac{1}{\pi+3}\right)\left(u W_{n}^{*}+s P_{n+2}^{*}\right) \text {. }
\end{aligned}
$$

Proofs are omitted. Although the first two have been well known in thenry for more than a century, the recurrence relations do not appear to have been used to calculate the magnetic properties of uxially symmetric systems until 1950. The first two formulas are given in the standard form. The last three conform closely to the arrangement that was actually used for fixed point machine coding. The reason for these special forms is discussed in Chapter 2. The recurrence formulas yield at the first application: $P_{2}=\frac{1}{2}\left(3 u^{2}-1\right), P_{2}^{\prime}=3 u, P_{2}^{*}=\frac{3}{2} u s^{2}, U_{3}^{*}=\frac{1}{2}\left(1-s^{3}\right) / u, W_{1}^{*}=\frac{4}{3} u s^{3}$.

The following equation is exact for all orders in the plane of the origin $(u=0)$, and is a good approximation at high orders for all angles:

$$
(n \gg 1), \quad U_{n+2}^{*} \cong-W_{n-2}^{*} \cong s P_{n} \text {. }
$$




\section{CHAPTER 2. MODIFICATIONS FOR USE WITH COMPUTERS}

The theory of Chapter 1 has been developed, and the equations have been written, in forms that need little change for practical use, with either a desk calculator or a programmed computer. ${ }^{10}$ They have proved to be highly efficient in solving a wide range of field, force, and mutual inductance problems, including design problems.

It is the purpose of this section to discuss certain points of numerical analysis, including the avoidance of difficulties that are capable of causing large rounding errors and the methods that were used to test the results. The procedures and numerical checks that are described here have proved useful with several computers, but the discussion refers specifically to the program as written for the IBM 7090. In the final section, this existing code is used to illustrate some general features that might be found in any versatile zonal harmonic computer program, but the details of the 7090 code are deferred for consideration in Chapter 3.

\section{Fixed and Floating Point}

All parts of the working program use single precision routines. The major portion is conveniently written in the Fortran interpretive language, which employs floating point arithmetic with a precision of 27 bits or about 8.1 decimal digits. It is necessary, however, when the highest precision is demanded, to use fixed point with its ability to retain 35 bits or 10.5 decimal digits, to compute both harmonic and logarithmic tunc. tions of the polar angles. Also, floating point conversion of non-integral input coordinates of the coil system from decimal to binary form, prior to their entry into the fixed point section, is avoided by so scaling the coordinates that they are expressed as integers. The fixed point section is coded in the FAP system (essentially the direct

\footnotetext{
${ }^{10} \mathrm{For}$ hand calculations the use of alternate-term recurrence formulas is convenient [Ref (1), p 1095]. The functions $U_{n}$ are tabulated in (2) through $n=17,8$ to 5 decimals, at interval 0.001 of the rosine argument, with second differences. There is also a brief pilot table of $W_{n}$ through $n=17,10$ decimals, at interval 0.1 .
}

machine language) and it includes the square root, the logarithm (for $q_{1}$ and $q_{2}$ ), and six recurrence routines based on Eqs. (41) to (45).

The modified functions $P_{n}^{*}, U_{n}^{*}$, and $W_{n}^{*}$ of Eq. (4) were defined expressly for fixed point machine calculation, since they remain between limits +1 and -1 for all orders and all polar angles. But to avoid overflow, the recurrence formulas also must be recast into forms such that no intermediate product or sum exceeds unity. Equations (43) to (45) satisfy this condition, but (41) fails to compute $P_{2}$ since $3 / 2 u^{2}$ can overflow. This equation must be divided through by $n+1$ and then rearranged like (43), with three terms on the right side, the first and last containing $u P_{n}$. The reciprocals of integers $(1 / n)$ are computed once for all and stored, at the start of the first pass through the fixed point subroutine.

\section{Calculation by Reverse Recurrence $(u<0.5)$}

Equation (44) for $U_{n}^{\star}$, alone among the recurrence formulas, presents a serious problem in the progressive loss of significant digits, if the argument $u$ is small." 1 The repeated multio plications by $1 / u$ amplify the early rounding errors intolerably. The normal use of this equation is therefore restricted to arguments greater than 0.5. For smaller arguments, $U_{n}^{\star}$ of highest required order is first computed from a power series in $2 u$. The functions of lower order are then found by successive iterations, using Eq. (44) in reverse, until $U_{3}^{*}$ is reachod. The necessary values of $P_{n}^{\star}$ are first of all computed in the usual way from $(43)$.

Eight options are provided, whose maximum order ranges in steps of 4 from 5 to 33 . Table 1 lists coefficients of the powers of $2 u$ for these series. The entire table is stored in the fixed point section of the IBM 7090 code, and the limiting order for any problem is selected by a data card elitry. As the order advances, there is a progressive loss of significant digits in the series sum, from the mutual cancellation of + and - terms. The magnitudes of the coefficients in

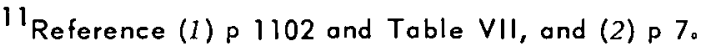


the working series require critical adjustment to minimize this loss while at the same time avoid. ing overflows. Each coefficient contains a factor $22010^{-p}$, with $p$ separately adjusted for each column. The series sum must then be divided by $(n-1) 2^{20} 10^{-p}$ since the coefficients were actually computed for $U_{n}=(n-1) U_{n}^{*}$. The divisor is listed at the foot of each column, together with the sum of coefficients (dividend) and the quotient. The quotient is the value of $U_{n}^{\star}(0.5)$ since for this argument all powers of $2 u$ are unity. For this limiting value the precision is least, and the progressive loss with advancing order is measured by the declining precision of the sums of successive columns.

\section{Derivation of the Cocfficients}

To compute the data for Table 1,12 calculations based on Eqs. (42) and (44) were carried through the 43 rd order, retaining 22 decimal digits. In place of (44), the corresponding formula in $U_{n}$ was used, and both this and (42) were rewritten with $w=2 u$ as the variable. The recurrence formulas were repeatedly applied, not to the successive orders of functions of a specific argument, but to the coefficients of the corresponding 40. term serics expunslons for the tunctions of the general argument. The initial functions were $s^{3} P_{1}^{\prime}(u)=\left(1-1 / 4 w^{2}\right)^{3 / 2}$, expanded to 40 terms by the binomial theorem, and $U_{2}=1$, both multiplied by $2^{20} \times 10^{-8}$. At each step, coeffirients of like powers were added before proceeding. The individual coefficients and the series totals were stored and carried through to the highest order. At eight selected points in the iteration, contents of the entire working storage hlork were shitted left one place to avoid loss of possibly significant figures, while to estimate the final precision the complete set of calculations was repeated with a shifted input decimal point.

The coefficients and series sums computed by this method are believed to be reliable to ten decimal digits even at the $43 \mathrm{rd}$ order. When used with the reverse recurrence routine, the coeffi-

\footnotetext{
${ }^{12}$ This port of the work was carried out on UNIVAC, using the direct machine language and a double precision fixed-decimal routine. The author is indebted to the computing center of the University of Pennsylvania for access to UNIVAC, while the cost of machine time was met in part by the National Science Foundation.
}

cients as tabulated should permit a working precision of four significant figurcs in the least favorable case, $u=0.5$ and $n=33$, taking account of rounding errors. The error in the series for highest order $(33,29,25$, etc.) is attenuated in the course of the iterations by successive mul. tiplications by $u$, followed by the addition of terms computed from (43). Since these added terms have full 10-decimul precision at the lowest orders, and never lose as many as two significant digits through cumulative rounding errors, the overall rate of attenuation of error is more than adequate to ensure the full usable precision at all lower orders. The functions $U_{n}^{*}$ are always less than unity, and their magnitudes decrease slowly with increasing $n$. They enter the expression for the source constants $q_{n}$ with the decreasing factor $1 /(n-2)$, and are finally multiplied in the field series by ascending powers of the ratio $R$ of polar radii, upon which the rate of convergence of the series princlpally depends. When the series is truncated, even one significant figure usually suffices for the highest order retained. These considerations would justify carrying the method of reverse recurrence at least to $n=41$, using single precision with a 10 or 11 digit muchine and restricting the range, as here, ro $u=0.5$.

\section{Limitation on Forward Recurrence}

The cumulative rounding errors in computing $q_{n}$ for thick coil sources are more serious when Eq. (44) is used in the forward direction. When $u=$ 0.5 , they may approach 4 or 5 percent at $n=33$, and they would be barely tolerable at $n=37$. To equalize the maximum errors of forward and backward iteration, the direct range of $\mathrm{Eq}_{\mathrm{q}}$ (44) might be ended at (say) 0.45, while to cover the vacated range Table 1 would be recalculated with $2.25 u$ as variable in place of $2 u$. In this way the muximum order could be raised to 41 or even to 45 . But the considerable labor is difficult to justify in view of the rather limited increment to the practical working range of the convergence ratio $R$. The probable extension can bc roughly judged from the nomogram (Fig。4).

Note that Eqs. (41) and (43), which suffice for loops and thin solenoids, and even (45) for the external constants of thick coils, permit good precision at much higher orders. It is in fact 
Table 1. Coefficients for Series Expansion of $2^{m} U_{n}^{\star} \times 10^{-p}$ in Powers of $2 u^{\dagger}$

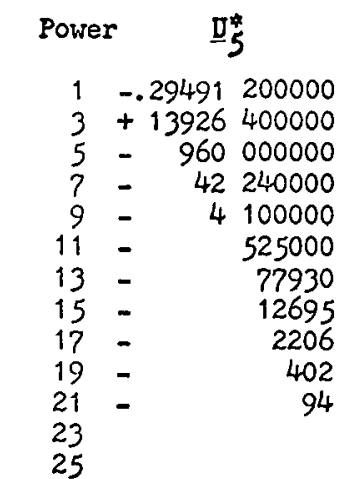

Divisor .41943040000

Sum $\quad-.16571758327$

$\underline{\underline{n}}_{\underline{n}}^{*}(.5)-.39510 \quad 1507$

Power U⿺辶⿸丆口

$1-.00368588220$

$3+5322013475$

$5-27152185607$

$7+58041292399$

$9-67949992644$

$11+47446503400$

$13-20478035660$

$15+5446901813$

$17-848885452$

$19+66342479$

$21-1507919$

23 - 29721

$25-\quad 1556$

27 - 135

29

31

33

Divisor .00209 715200

Sum +.00024331651

$U_{n}^{*}(.5)+.116022353$ $\underline{v}_{9}^{*}$

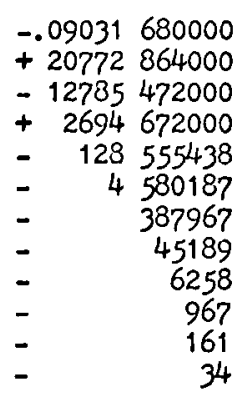

.08388608000

$+.01516307798$

$+.180817580$

눈 25

$-.0001 \% 590303$

$+1115708114$

- 7601887848

$+24031836588$

- 42483727912

$+46266199492$

- 32715132626

$+15395937586$

- 4831023151

$+\quad 987053850$

- 123161362

$+\quad 8017799$

- 153395

- $\quad 2617$

- 130

.00025165824

$+.00001074084$

$+.042630246$
Uै 3

$\begin{array}{rr}-.01691289600 \\ +\quad 9241689600 \\ -14458544100 \\ +\quad 9572806100 \\ -\quad 2970278695 \\ +\quad 402058236 \\ -\quad 14066006 \\ -\quad 39636 \\ - & 28336 \\ - & 2874 \\ - & 356 \\ - & 59\end{array}$

.01258291200

$+.00081945273$

$+.065124252$

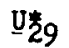

$-.00006135019$

$+\quad 193014518$

- 1807265989

$+7907673052$

- 19579867493

$+30399445643$

- 31431361836

$+22422336217$

$-11238005508$

$+3970901413$

$\begin{array}{r}-\quad 977276898 \\ \hline\end{array}$

$+\quad 162024492$

- 16855453

$+\quad 935181$
$+\quad 16$

$\begin{array}{rr}- & 15527 \\ - & 244\end{array}$

.00002936013
-.00000403502

-.00000403502
-.137432099

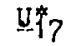

$-.02625480000$

$+26157560000$

- 76223154150

$+99434709563$

- 68095850856

$+25897480839$

- 5373590298

$+\quad 535152864$

- 14694135

- $\quad 342245$

- 20687

$\begin{array}{rr}\text { - } & 1831 \\ - & 232\end{array}$

.01677721600

$-.00308231170$

$-.18372 .0094$

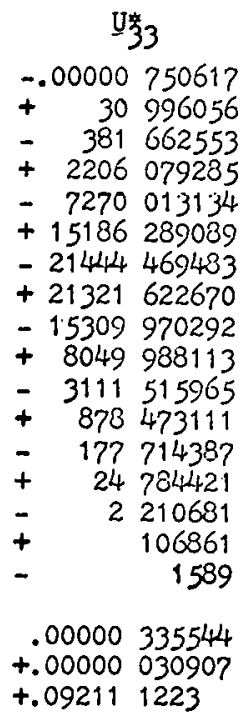

TThe digits are properly aligned, but coefficients after the first order are multiplied by $10^{11}$, that is, the decimal poinl and leading zeros are suppressed.

entirely practical to use higher orders if it is necessary to work somewhat closer to a thin winding. Analysis of the integer factors in the source Eqs. (32-34) and (37-39) for coil, solenoid, and filament sources suggests that the rate of convergence of the field series should decrease appreciably in the order named. The inference is confirmed by experience.

\section{Monitoring the Convergence}

Computing time can be saved by truncating the field series when a convergence test is satisfied, but a simple test of magnitude cannot be safely applied. (See the discussion of convergence in Chapter 1.) Each series term is the product of a source harmonic and a field harmonic. The products oscillate within predictable limits whose smoothed rate of decrease depends mainly on the convergence ratio $R$. The short-range variation of signs and magnitudes, though amenable to analysis, may conform to no very obvious pattern. Two or three successive near-zero terms may be followed by others of appreciable magnitude, even though there is no risk of eventual divergence.

For this reason a running sum of the absolute magnitudes of the last six terms is carried, and 
the field series is stopped when this sum falls below some value that is preset on a data card. The running sum and the order at which truncation occurs are printed. There is also an optional print of the initial terms, or of the final terms, or both. A study of the final terms supports the assumption that the running sum affords a conservative estimate of the maximum truncation error.

\section{The Logarithmic Functions}

The lowest-order source constants $q_{1}$ and $q_{2}$ for thick-coil sources contain logarithmic factors. (Eq. 34.) Where a coil is far from the orlgin and the linear dimensions of its section are comparatively small, two or more significant figures can be lost in the summation of + and $-q$-constants of nearly equal magnitudes over its four sources. Logarithms were at first computed in the Fortran section of the 7090 program by the standard routine, which is subject to errors of 3 in the 8th significant decimal digit. Initial conversion of input data from decimal to binary may introduce errors of one unit in the same place. Unexpectedly large discrepancies appeared on comparing sets of values of $B_{z}$ computed for a fixed net of field points but from two or more origins, especially when field contributions from distant coils were significant. Eprops were found, of the order of 5 purts per million, that were nearly constant over the whole net for a given origin, but were random from origin to origin. The difficulty was traced to the first term of the field series, that is to $q$, .

The discrepancies were reduced to one or two parts in $10^{7}$ by recoding parts of the program in fixed point and incorporating them into the FAP suhroutine, which resulted in a gain of nearly 8 significant bits. The altered code introduces an arbitrary scaling factor RS (usually a power of 10) by which all linear coordinates of a system are multiplied so that they appear as integers on the data cards. In the FAP section, the source coordinates $z_{j}, \rho_{j}$ of each source enter at once a routine that preserves only their ratio, while converting the larger quantity to a fraction with 35 significant bits, that is, one whose mag. nitude is at least 0.5 but less than 1.0. The normalizing factor is discarded. The ratio is used to compute the sine and cosine of the polar angle, from which all remaining angular functions are calculated in fixed point.

The logarithm is computed once only for each source plane, or twice for a complete coil. That is, in place of the difference in logarithms of $(1+s) / u$ for the two sources, the logarithm of the ratio is computed, using a 4 -term Hastings approximation that is good to $2 \times 10^{-10}$. Constants nre summed over the two source planes of each coil before conversion to 27-bit flanting point; while removal of the factor RS and all further calculations involving linear magnitudes are done in the Fortran sections.

\section{Zonal Harmonic Section Expansion}

Concurrently with the changes discussed in the last section, tests were made in which the constant $q_{1}$ for a thick coil was derived from that of its central filament by a series expansion. This was done by writing a two-dimensional Taylor's corioc in powers of $R$ and $n$, in which the zero-order term is given by $\mathrm{Eq}_{\text {. }}(32)(n=1)$, and higher orders ore derived from Eq. (3) and related general expressions for partlal derivulives of the zonal harmonics. $B$ and $D$ are respectively the axial breadth and radial depth of the cross. section, normalized by dividing by the mean cylindrical radius $\rho_{0}$.

In this way an expression was obtained for the general order $q_{n}$, but $q_{1}$ unly will be cunsidered here, since higher orders were nol used in this work. Residual errors are of the sixth order, since the second and fourth are retained, and odd orders vanish by symmetry.

When $n=1$, the general expression reduces to

$$
\begin{aligned}
q_{1}=N^{\prime \prime} B D \rho^{2} r_{j}^{\star-1} s^{2} & {\left[1+\frac{1}{12}\left(B^{2}-D^{2}\right) s^{2} P_{3}^{\prime}+\frac{1}{60} D^{2}\left(P_{3}^{\prime}-1\right)\right.} \\
& +\frac{1}{240}\left(B^{2}-3 D^{2}\right)\left(3 B^{2}-D^{2}\right) s^{4} P_{5}^{\prime}+\frac{1}{1080}\left(5 B^{2} D^{2}-3 D^{4}\right) s^{2} P_{5}^{\prime} \\
& \left.-\frac{1}{8640}\left(40 B^{2} D^{2}-33 D^{4}\right) s^{2} P_{3}^{\prime}\right] .
\end{aligned}
$$


Compare Eq. (32). The expression enclosed in brackets in $\mathrm{Eq}_{0}$ (47) is the ratio of $q_{1}$ for the coil to that for an ideal filament of $N^{\prime \prime} B D \rho^{2}$ turns at the center of the section. Equation (47) is an approximation that is similar in principle to that of Lyle's well known formula, ${ }^{13}$ which gives much the same result as the first line of (47). Inclusion of the fourth-order terms in the second and third lines greatly improves the precision.

Although this expression was coded entirely in 27-bit floating point Fortran its use leads, as expected, to errors even smaller than those of the 35-bit fixed point logarithmic routine, in the case of coils very far from the origin. At intermediate distances, the two methods are equally precise, but of course when coils of large section are placed so close to the origin that terms in $B^{6}$ and $D^{6}$ are significant, the calculation by logarithms is the more precise. Since in practice the modified logarithmic code proved to be adequate, the method of section expansion was dropped, and it was never coded for $q_{2}$ or higher orders. However, hand calculations established the validity of the general form for odd orders through $n=7$.

\section{Numerical Checks}

The systematic character of the theory and of the machine programs makes it easy to devise internal checks, to test the self-consistency and precision of the overall procedure. Three examples of somewhat different types may be mentioned. A very severe test is the agreement of results calculated from two or more origins. Hundreds of such comparisons for field components, forces, and mutual inductances have shown agreement to seven significant figures, though the high-order source constants may change

${ }^{13}$ Lyle's approximation is ideally simple and surprisingly accurate when the cross section is square. It then concentrates all ampere turns in a single filament, but at the rootomean-square rather than the mean radius. If the extreme radii are $\rho_{1}$ and $\rho_{2}$, the squared equivalent filoment radius is $\rho_{F}^{2}=1 / 3\left(\rho_{1}^{2}+\rho_{1} \rho_{2}+\rho_{2}^{2}\right)$. This approximation removes all second-order errors of finite section (i.e., terms with the factor $B^{2}=D^{2}$ ) from the series exponsion of $q_{n}$ or $p_{n}$ of any order $n$. When the section is not square, two loops are required, and the error Inereases rapidly with the larger dimensiun of the section. by many orders of magnitude. Significant discrepancies occur only when the series referred to one or both origins has run through to the maximum order. Such discrepancies are comparable in magnitude to the running sum referred to above, and to the errors predicted by the nomogram. A second kind of internal test is to compute the constants of a thin cylindrical coil from the equations for both solenoid and coil source types. A third is to compare computed values of $\partial B \rho / \partial z$ with the computed effect of finite $z$-increments on $B \rho^{\prime}$ or of $\rho$-increments on $B_{z}$.

Several hundred comparisons made on the field of the DCX-2 system, with $B_{z}$ and $B_{\rho}$ values computed by Downing's code, ${ }^{7} z$ showed agreement within one or two units in the 7th significant figure. More recently, the author's elliptic integral code ${ }^{7}$ was used to check values of $B_{z^{\prime}}$ $B, A, F$, and $M$, with comparable results. Both of the comparison codes are slower than the zonal harmonic program, probably by about one order of magnitude.

The harmonic functions as computed in the fixed point section using the recurrence formulas, were checked to high orders against values previously calculated on other machines. The precision of the logarithmic functions and of the values of $q$, computed by section expansion from a central filament were checked by analysis of fourth differences, as follows.

Sets of logarithms were calculated for the successive terms of several geometric series, over the basic binary range 0.5 to 1 , using a constant ratio $1+a$. In theory, all differences except the first should vanish. The entire calculation, including the fourth difference routine, was carried out in fixed point in the FAP section, and the terminal fourth differences alone were printed out after decimal conversion. Values of $q_{1}$ through $q_{5}$ for several single thick coils were computed for origins arranged in 8 or 10 sets of 12 each. The sets were far apart, but origins were equally spaced at a small interval within each set. Fourth differences were computed within each set. Here $q$, was computed by the revised logarithmic code as described above, by a straight Fortran program, and by section expansion. By these tests it was verified that both the logarithm itself and the complete routine in which it is used result as expected in a gain of two significant decimal digits when compared to 
a straight Fortran code. It seems also that there is no need to resort to the method of section expansion, except perhaps in a high precision investigation of the field of an infinite array of coils.

\section{A Flexible Zonal Harmonic Computer Program}

A few general suggestions based on experience in organizing the existing 7090 program may make it easier to adapt the method developed in this report for use with other machines. The details that are needed for the effective use of the actual program are discussed in Chapter 3.

The currently completed code includes most of the range of calculations that is discussed in Chapter 1. The force and mutual inductance, however, are restricted to coaxial systems. Also, though the remote source constants $p_{n}$ are computed for all systems, the remote field components of Eqs. (18) to (21) have not been coded. Disk or pancake coils are not now included. The missing parts might be added with very little labor, since all the harmonic functions that they require are computed in the existing routines.

All data cards conform to a single pattern that provides four fields for small integers and six tor decimal data. Flexibility of operations resides in the many switching options, exercised by single digits in the integer fields. These control the reading of data, the sequence of operations including at times the calling of subroutines, the quantities to be computed, and the extent and form of output data to be printed or punched. To simplify the input, the most frequently used option is in most cases a blank field.

Several versions already exist for certain subroutines. To provide for additions and special small problems or tests, the sequence options also include calls for several dummy subroutines. A reduced optional form of the main program (abstract) retains only a card read and a set of sequence options. These three features permit the solution of a wide range of problems with a minimum of recoding. Essential parts of the complete system are listed in the following paragraphs, with the hierarchy of control in reverse order.

The fixed point subroutine uses the appropriate recurrence formula to compute any required set of harmonic functions of the polar angle for a single source. As noted above, it also computes the logarithmic source functions $q_{1}$ and $q_{2}$ for each pair of coplanar sources and sums them for a complete coil. It is called by two other subroutines, which complete the calculations for the source equations and the field equations re. spectively.

The source routine computes either $q_{n}$ or $p_{n}$, and on demand also their rates of change with respect to source coordinates, for a single element of the system. This is calculated as a thick rectangular coil if the axial breadth $B$ and radial depth $D$ are finite, as a solenoid if $D=0$, and as a loop if $B=D=0$. This subroutine supplies the proper number of sources $(4,2$, or 1$)$ and sums the constants over all sources. The rates of change üre fur use in sulviny design problems.

One field subroutine reads in one or more sets of origins (by ordinal number), parameters for one or more nets of field points to be associated with each origin, and specifications of the field components that are required, together with output print options. Another version accepts coordinates of a single field point or of a complete net of points, and automatically computes from the nearest origin for each point. This is the proper field routine to use when tracing ion orbits. A third field subroutine computes mutual inductances and axial forces between coaxial systems. Each of the three versions requires a complete set of source constants $q_{n}$ for each origin, previously summed over the entire system and stored, while the third requires also the $e^{\circ}$-constants of the secondary system.

The main program controls the sequence of operations. It reads in the geometry of the current system and nbsrissns of one or more sets of equally spaced origins, calls for the source constants of each element in turn, sums the constants over the entire system for each origin, prints and stores the sums, and exercises various options under control of the data cards.

It is clear from a study of the field equations that significant sections of coding can be common to two or more field components. Similar economies can be made in the source subroutine, with respect to different source types, and also in the calculation of derivatives. [See Eqs. (28$31),(35-36),(40)$. 


\section{CHAPTER 3. ZONAL HARMONIC CODES FOR THE IBM 7090}

Chapter 3 assumes that the reader has a working knowledge of 7090 Fortran. The existing 7090 codes comprise a main program, a program abstract, and eight subroutines, some of which have several versions. Only single precision routines are used, though one major section that is called by all the others is written in the FAP language, mainly because it requires the 35-bit precision of fixed point arithmetic. All other parts are coded in Fortran. Identical sets of COMMON and DIMENSION cards are used in all Fortran source decks, and all variables are transmitted from one section to another through COMMON storage.

All data cards conform to a standard pattern with eleven available fields $(6 \mathrm{H}, 413,6 \mathrm{~F} 9.4)$. The four integers are available for counts or limits, and to specify switching options. The six decimal fields may be used for numerical data, or to select various options. Table 3 summarizes the uses of data fields on the five cards that are read in by the main program and by subroutine ZEBRA, which are treated more fully in the next two sections. The first column of the table identifies the card by type, while the second column gives the number of the Fortran statement that reads in the card. Of the 46 fields listed, about two-thirds have zero options, which in practice means a blank field on the card. Wherever possible, the blank is assigned to the most common option. Parentheses in the table identify fields that may be blank, while double parentheses enclose dummy symbols that are always blank. The first Hollerith spoce of some data cards is used to control line spacing or paging of the output print. Thus a 1 is usually entered in column 1 of START and ZEBRA cards, to begin a page, while the remaining cards begin with 0 . The remaining five Hollerith spaces of any card may carry some brief code to identify either the card or the problem (in place of the letters START, etc., of Table 3).

Special data forms and a master card or template have been made up to facilitate the preparation and checking of the data cards. As each card is read in, it is reproduced at once on the output tape. With these aids it is easy in spite of the many options to write and check a set of data cards after one or two problems have been run.
Most options will be blank, and with few exceptions, it is simplest to omit decimal points in the numerical fields.

A considerable variety of problems may be solved by suitable combinations of the five types of data card. The flow of the program, including the reading and processing of data and the sequence of subroutines, is controlled by the switching entries on the cards, either in the main program or in certain subroutines, notably ZEBRA. Thus it is possible without changing the main program to add sections of coding, to make special tests, or to introduce major variations or new problems, by using new card se. quences and changing the content and sometimes also the function of one or more of the named subroutines. Several of these are normally dummies for any particular problem.

The following paragraphs outline briefly the functions of the most commonly used sections of Fortran coding. Discussion of the FAP section, and of the remaining Fortran subroutines, is deferred while three other sections of the text are developed. The first two describe some details of the COMMON storage area, and of the five types of data card that are required by the main program and by subroutine ZEBRA. The third describes the complete sequence of cards for solving two typical problems.

The main program abstract (MPA) may be conveniently used for tests, and for occasional new problems or variations. It includes only the standard COMMON and DIMENSION lists, plus a set of switching options. It reads in a START card, as shown in the first line of Table 3, initially and again at each return from any sub. routine. This card transfers, as shown under the first entry IX, to any of six subroutines or to EXIT. It provides nine additional fields to carry data or switching options to the selected subroutines. Note that all ten fields of the START card load into COMMON storage locations. For increased flexibility, the meaning of some of these symbols may be safely changed to fit new variants of the subroutines.

The main program (M.P.), besides performing the control function just described, carries out the first stage of problem solving as discussed in 
Chapter I, namely the calculation of source constants $q_{n}$ or $p_{n}$ from the source equations.

It reads in the geometry of one or more complete systems or subsystems, and the abscissas of one or more sets of origins that are to be used. For each origin (subscript $J$ ) it computes, prints, and stores in $Q S(J, N)$ a set of source constants for the system or subsystem, and prints also if required the contributions from separate elements of the system, with or without the rates of change of such contributions with respect to $z$ or to $\rho$. It calls FINDQK for most of the actual calculotion.

FINDQK computes $q_{n}$ or $p_{n}$ up to the maximum order MAXN, for a single element of the system, referred to a single origin. Each element is identified by a subscript $K$, hence $Q K$ in the title. An element of the system may be a loop, a thin solenoid, or a rectangular coil. Either of the last two may be semi-infinite. Optionally, FINDQK can also compute derivatives of the source constants. This subroutine reads no cards and prints no results. It calls the FAP section to compute functions of the source angles.

ZEBRA (1) (a skewed mnemonic for $B Z, B R, A$ ) employs the field equations to carry out the second stage of solution as defined in Chapter 1. It reads in one or more sets of origin subscripts $(J)$, with the corresponding abscissas, and one or more nets of field points to be referred to each set of origins, also information about the field components that are desired and certain specifications of output print options. It refers to the source constants previously stored in $Q S(J, N)$, calls the FAP section to calculate functions of the field angles, computes and prints the field information required. On demand, it also stores in BN sets of values of a specified field component, for each point of a prescribed field net, for each of a given number of subsystems. These values are for use in an optimization routine. (See BEST).

COMMON STORAGE. THE MAIN PROGRAM (M.P.)

The COMMON storage area of 4714 cells is mapped in Table 2. Horizontal spaces divide sections that are treated as separate units in PDUMP operations initiated by the instruction CALL OPEN and controlled by the $X$ field of certain data cards. (See below.) References to the source constants $q_{n}$ in the last column of the table include $p_{n}$ as an alternative. These remote source constants are computed in place of $q_{n}$ when IX $=1$. DIMENSIONS of the variables are listed in the first column.

The reader will need to refer constantly to Tables 2 and 3 when reading this and the following sections. It will be assumed that many entries in the tables are self-explanatory. Table 3 is intended to be the routine source of all information that will normally be needed to assemble a data card deck.

\section{The START Card}

IX, K. - A negative entry in lix causés an limmediate transfer to one of several subroutines, as may be seen from Table 3. A blank or 1 starts a pass ihrough M.P. On euch puss llie pruyiuin cuiliputes, stores, and prints a set of source constants for a complete system or subsystem, and then either transfers to ZEBRA or calls for a new START card, depending upon IB (q.v.). A blank in IX produces $q$-constants, while $\mathrm{IX}=1$ generates $p$-constants. Each pass through M.P. requires a START card, $K \mathrm{~K}$-cards, and one or more ORIGN cards, one for each set of equally spaced origins. $K$ is the number of elements, or pairs of elements, in the system or subsystem, and the minimum number of cards is $K+2$.

J. The J- or Origin Count. - Stored information for each origin includes its abscissa $O(J)$, the minimum or maximum polar source radius RX2(J) that determines its sphere of convergence, and a set of source constants $Q S(J, N)$ that may be either $q_{n}$ or $p_{n}$. A flexible control over the subscript $f$ allows such data to be computed and stored in a variable sequence up to a maximum value $J=M X J$ of 24 . The stored data may be accumulated during several passes through M.P., or it may be read, in whole or in part, from cards punched by a previous program. Data to which different subscripts $J$ are assigned may belong to several systems or subsystems, and may include $q$-constants with minimum source radii for some origins and $p$-constants with maximum source radii for others. See for example the dis. cussion of TEST (M), and of the second problem below.

Subroutines PUNCH and READ, which permit intermediate storage of problem data on cards, always begin at $J=1$ and end at $J=M X J$. PUNCH 
Tabla 2. Guide to Common storog*

\begin{tabular}{|c|c|c|c|c|c|c|c|c|}
\hline \multirow{2}{*}{$\frac{\text { Dimensions }}{8,100}$} & \multirow{2}{*}{$\frac{\text { Subscripts }}{\text { I, M2 }}$} & \multirow{2}{*}{$\frac{\text { Torol }}{800}$} & \multicolumn{2}{|c|}{ Begin } & \multirow{2}{*}{$\frac{\text { Symbol }}{\mathrm{BN}}$} & \multicolumn{2}{|c|}{ End } & \multirow{2}{*}{$\frac{\text { Explanation }}{\text { Dato fst optinization }}$} \\
\hline & & & 66 & 310 & & 67 & 747 & \\
\hline 40,34 & $K, N$ & 1360 & 67 & 750 & OK & 72 & A57 & $a_{n}$, se sorate =ails \\
\hline 4,34 & $L, N$ & 136 & 72 & 470 & 0 & 72 & 877 & $q_{n}$, sosazole sourees \\
\hline 4,18 & $L, N$ & 72 & 72 & 700 & DA & 73 & $\mathrm{COZ}$ & Derivocives, seporate surces \\
\hline 16,18 & K, N & 288 & 73 & 010 & $\mathrm{DA} 2$ & 73 & 447 & Rodial ferivatives of ? \\
\hline 16,18 & $K, N$ & 288 & 73 & 450 & DAI & 74 & 107 & \\
\hline 16,18 & $K, N$ & 288 & 74 & 110 & 022 & 34 & 547 & Axial derivatiess of $q_{q}$ \\
\hline \multirow[t]{3}{*}{16,18} & $\mathrm{~K}, \mathrm{~N}$ & 288 & 74 & $5 \leq 0$ & $D Z I$ & 75 & $2 \times 7$ & \\
\hline & $\mathbf{K}$ & 16 & 75 & 210 & IA & 75 & 227 & Switch, rodial dorivotives \\
\hline & K & 16 & 75 & 230 & 12 & 75 & 247 & Switch, axiol d'erivative: \\
\hline \multirow[t]{20}{*}{24,33} & $J, N$ & 792 & 75 & 250 & as & 16 & 677 & $q_{n}$ lor sesrom, soch orig $n$ \\
\hline & $J$ & 24 & 76 & 700 & $\mathrm{R} \times 2$ & .6 & 72.7 & Min or mox polaz radius. \\
\hline & $j$ & 24 & 76 & 730 & 0 & .6 & $7: ?$ & $z$ coordi note of origin \\
\hline & & & & & 010 & 76 & 760 & $q_{1}$ for $x$ entral origin \\
\hline & & & & & $\mathbf{B Z I}$ & 76 & $76:$ & $B_{z}$ for e-ntrol origin \\
\hline & & & & & RS & 76 & 762 & Scale fartor, input goonetry \\
\hline & & & & & RO & 76 & 765 & Unit or reference lengtt $\bullet_{0}$ ) \\
\hline & & & & & $M \times J$ & 7s & 764 & Limiting volue of $\mathrm{J}$ \\
\hline & & & & & $M 1$ & 75 & 765 & Switch (sovaral usos) \\
\hline & & & & & 1 & 73 & 765 & Count of sub-systems \\
\hline & & & & & $x$ & 75 & 767 & Switch to PDUMP \\
\hline & $k$ & 40 & 76 & 770 & Co & 7 & $03 ?$ & Current donsity \\
\hline & $\mathbf{k}$ & 40 & 77 & 040 & D & $7:$ & $10 ?$ & Rodiol fepth of coil soction \\
\hline & $\mathbf{K}$ & 40 & 77 & 110 & $\mathbf{B}$ & $7:$ & 157 & Axial brosdth of coil soet on \\
\hline & $\mathbf{k}$ & 40 & 77 & 160 & A0 & 7 & 227 & Mean radiol coordinate \\
\hline & $\mathbf{K}$ & 40 & 77 & 230 & zo & 7 & 27 & Meon oxisl coordinato \\
\hline & & & & & $J$ & 77 & 300 & Origin cornt \\
\hline & & & & & $\mathrm{x}$ & 77 & 301 & System aliement (coil) court \\
\hline & $\mathbf{L}$ & 4 & 77 & 302 & $\mathbf{A}$ & 77 & 305 & Rotiol source coordinato \\
\hline & $\mathbf{L}$ & 4 & 77 & 306 & $\mathbf{Z}$ & 77 & 311 & Axial souxe coordinate \\
\hline \multirow{15}{*}{$\cdot$} & & & & & $M \times D$ & $\pi$ & 312 & Limiting coder, derivatives \\
\hline & & & & & IX & 77 & 313 & Moin socusence switch \\
\hline & & & & & $R X$ & 77 & 314 & Current min or mox poles \\
\hline & & & & & OR & 77 & 315 & $z$ esordince of eurrent oris in \\
\hline & & & & & WN & 77 & 316 & \\
\hline & & & & & SPN 1 & 77 & 317 & Switches for FAP options \\
\hline & & & & & PN & 77 & 320 & \\
\hline & & & & & $A C$ & 77 & 321 & Cyl. rodius \\
\hline & & & & & $2 \mathrm{C}$ & 77 & 322 & Axisl coordinate \\
\hline & & & & & $\mathrm{U}$ & 77 & 323 & (input to FAP) \\
\hline & & & & & 5 & 77 & 324 & Lsine \\
\hline & $\mathbf{N}$ & 43 & 77 & 325 & RCP & 77 & 377 & Reciprocal of integers \\
\hline & & & & & L & 77 & .400 & Source cousts, 1 to 4 \\
\hline & & & & & MAXN & 77 & 401 & Limiting order, 5 to 33 \\
\hline & $n$ & 48 & 77 & 402 & QF & 77 & 461 & Functions +1 ongle (from FAP) \\
\hline
\end{tabular}


Toble 3. Ourline of Dose Card Fields

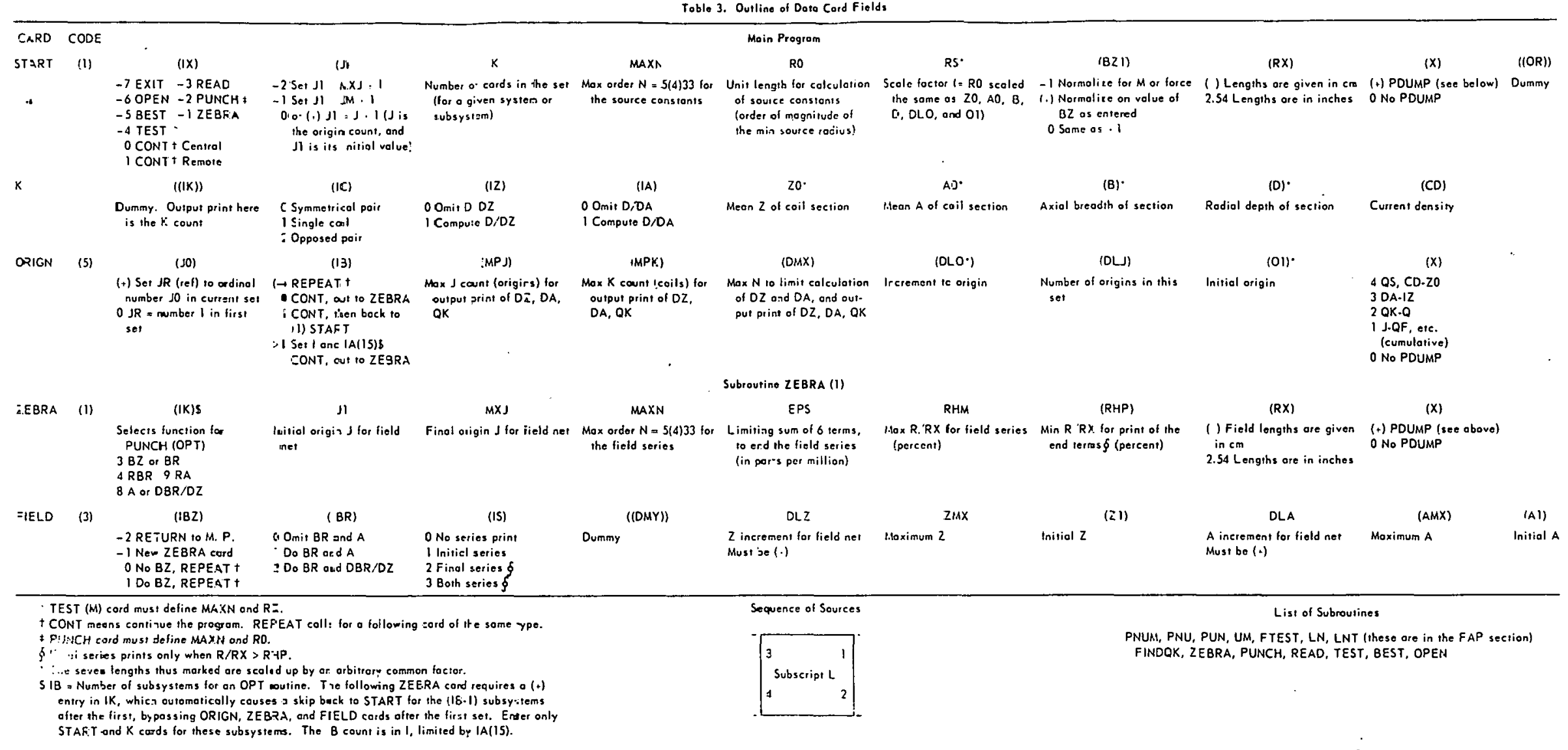


reads the value of MXJ from COMMON, while READ obtains it from the first card. Data computed in any one pass through M.P. are stored with subscripts in ascending order, in successive sets each of which is generated by a single ORIGN card and corresponds to a set of equally spaced origins. The J-count proceeds from $\mathrm{Jl}$ to a current maximum JM, both of which occupy cells in the M.P. storage area.

At the end of each such pass, MXJ in COMMON is replaced by $J M$ only if $J M$ is the larger. $M X J$ is thus an overall maximum count, cumulative for an entire problem and in fact for a set of problems. It can be reset only by the entry $J=-1$ on the START card. The count starts at $J l=M X J+$ $l$ if $J=-2$, at $J M+1$ if $J=-1$, and at $J+1$ if $J$ is blank or $(t)$. The blank is regarded as "normal," that is, the count normally begins at $J=1$. Examples of other options are given in the section on problem setup.

MAXN. - This is the limiting order for $q_{n}$ or $p_{n}$. It must always lie in the range 5 to 33 , and it is further restricted if $q$-constants are to be computed for thick coils. It must then be one of the 8 integers, $5,9, \ldots, 33$ in steps of 4 within this range, since the reverse recurrence routine for the source functions $U_{n}^{\star}$ must start with one of the 8 power series of Table 1. The higher values of MAXN are necessary only when fields must be computed close to the windings, or when mutual inductance and forces are calculated for coils close together. (See the nomogram, Fig. 4).

RO, RS, RX. Scaling and Units. - RO is the unit or reference radius $r_{0}$ of Chapter $\mathrm{I}$. It should be roughly of the same order as the radius of the sphere of convergence, that is the minimum polar source radius of a central field or the maximum source radius of a remote field. Otherwise the constants $q_{n}$ or $p_{n}$ of high orders may assume inconveniently large or small numerical values, as may also the high powers of the normalized radii in the field equations. Evidently a mismatch by a factor of 10 can generate extreme magnitudes of the order $10^{\mathrm{MAXN}}$ and $10^{-M A X N}$. Though this may not of itself lead to errors of calculation, such magnitudes are incompatible with the F-type decimal conversion which has been found convenient for part of the output print.

RS is just RO scaled up by the arbitrary factor $\mathrm{RS} / \mathrm{RO}$ (usually a power of 10) which is common to the entire input geometry, that is to $Z 0, A O$, $B, D, D L O$, and 01 , and which is divided out in FINDQK, before $q_{n}$ or $p_{n}$ are calculated. It may be unity, or it may be so chosen that the input geometry can be described by a set of integers (which may safely include mixed numbers that contain only exact binary fractions). This precaution is necessary only in critical cases, when it is desired to avoid random con. version errors that may lead to discontinuities of the order of some 5 or 10 parts per million in the fields of extended systems, as computed from multiple origins. The reasons are discussed in Chapter 2, and are considered again below when the FAP section is described.

$R X$ is blank if the system geometry is expressed in centimeters, 2.54 if it is measured in inches. In either case, though the output print is based on the same unit of length as the input for the geometry of the system and of the field, the magnetic units are based on the centimeter and the gauss. That is, the vector potential is in gauss $\mathrm{cm}$, flux in gauss $\mathrm{cm}^{2}$ or maxwells, $\partial B \rho / \partial z$ in gauss $/ \mathrm{cm}$, etc.

BZ1. Normalization. - The scaling factors for field components, mutual inductance and forces all depend on the scaling or normalizing factor for the source constants, which is determined by the BZI entry on the START card and the J0 entry on the ORIGN card.

The special code entry $B Z 1=-1$. generates directly the constants $q_{n}$ and $p_{n}$ of Eqs. (32-34) and (37-39), without special scaling. The fields $B_{z}$ and $B_{\rho}$ are then computed in gauss (or in kilogauss. if currents are in kiloamperes), $M$ is in henrys and $F$ in newtons. This is the correct normalizinq option when computing absolute field values from known currents, ui whon the constants are to be used in TEST(M) to compute $M$ and $F$. Any other magnitude assigned to $B Z 1$ serves to define $B_{z}$ at the origin selected by the Jo entry (q.v.), and the source constants are scaled accordingly. For convenience a blank BZ 1 field is equivalent to +1 ., which covers the very common case in which all field magnitudes are scaled to the arbitrary value $B_{z} \equiv 1$, assigned at any selected origin.

X. Control of P.DUMP. - A controlled P.DUMP can be made available at any point in the program or subroutines, by inserting the instruction CALL OPEN. It can also be initiated without this instruction, by the entry IX $=-6$ on a START card. 
It operates only when $X$ is set equal to 1., 2., 3., or 4. Each option dumps the storage areas of $M P$ and of subroutines FAP, FINDQK, and ZEBRA, in addition to COMMON storage J through QF, while options 2 to 4 include additional areas of COMMON as listed under $X$ in Table 3 . The four options are limited by separate counts in OPEN, in such a way that not more than four dumps can be obtained from any one value assigned to $X$, at each loading of the machine.

\section{The K.Cards}

IC. - One K-card is required for each element or matched pair of the magnetic system. If IC is blank the loop, solenoid, or coil is duplicated in memory as a symmetrical pair, to which sueeessive subscripts $K$ arc assigned, $K=1$ signifies a single element, $I C=2$ an opposed pair. When the last $K$-card has been read in, the total number of elements, counting each prir oc 2, is entered in MXK. This may not exceed 40 .

IZ, IA, DMX. - In addition to the source constants of any given element or pair, $z$-derivatives of the constants are computed if $I Z=1$, and $\rho$ derivatives if $I A=1$. Such derivatives are often useful as an aid to system design. The limiting order DMX for such derivrtivon rimul cxceed ly, and of course cannot exceed MAXN. The IZ option is limited to elements whose K-subscript is 16 or Inwer, as for exumple, the tirst 8 pairs. The IA option is restuicted to K-values of 13 or less, since memoly cells $I A(14)$ through (16) are preempted.

Z0, AO, B, D. - These coil dimensions are explained in Table 3. All nre expressed to the same scale as RS. If $D$ alone is blank, the ele. ment is computed as a thin solenoid, while for a loop both $B$ and $D$ are blank. Finully, the entry $B=-1$ causes the program to generate constants for an infinite thin or thick solenoid extending from $z=Z 0$ to plus infinity.

CD. ... If CD is blank, it is taken to be +1 . This is either a current, a linear current density, or a space current density, depending on whether the element is a loop, thin solenoid, or thick coil. The unit of length for current densities is the $\mathrm{cm}$ if $R X$ is blank, the inch if $R X=2.54$. Turn counts replace currents and turn densities replace current densities, if mutual inductances alone are to be computed.

\section{The ORIGN Card}

J0. - One card is required for each set of equally spaced origins. The program assigns subscripts $J$ to the origins in the order of entry, beginning with $\mathrm{Jl}$ as described above under J-count. If JO is blank, the origin of reference for normalization of system constants (subscript JR) is identified with the first origin of the first ORIGN card. $J 0$ is also blank when $B Z=-1$, since then normalization does not occur. $A(t)$ value of $J 0$ on any card resets JR to the ordinal number $J 0$ of the set that is specified by that card.

IB. - The field IB is a sequence switch. If negative, it calls for a new ORIGN card, to read in a new set of equally spaced origins before proceeding. When it is non-negntive, the program goes on to compute source constants for all origins. If IB is zero, or greater than $I$, the sequence then leads out to ZEBRA, but if $I B=1$, the main program reverts to stop (1) and reads in a new START card.

Any value of $\mathrm{IB}$ from 2 to 8 causes subroutine ZEBRA to print and punch out on cards IB separate sets of data representing the contributions to a chosen field component that arise from subsystems or individuol elements of a generating system. Such infarinulion is needed for any optimization routine that must odjust currents in the IB independent subsystems, in order to satisfy some cundition imposed on the combined field. The fiell component to be computed $\left(B_{z}, B_{g}, p, A\right.$, ote.) is specified by IK on the following ZEBRA card (q.u.). The network of field points is defined once only, since it must be the same for all subsystems,

Switching for this case is automationlly controllerd by the IB entry, which suppresses the reading of IB-1 sets of ORIGN, ZEBRA, and HIELD cards, though IB complete passes are made through MP and ZEBRA. Each pass after the first requires only a START card and a set of Kecords, to describe the geometry of a new element or subsystem.

MPJ, MPK, DMX. - These fields limit respectively the number of origins, the number of elements of the generating system, and the order, for which separate sets of source constants, with their axial or radial derivatives, are printed out. Source constants only are printed for any element unless IZ, IA or both are non-zero for that element, while if MPJ or MPK is blank, only the 
source constants for the complete system are printed.

DLO, DLJ, 01, X. - The first three fields define a set of DLO origins on the axis, beginning at $z=01$, with constant interval DLJ. The Xfield has already been discussed.

\section{SUBROUTINE ZEBRA (1)}

\section{ZEBRA and FIELD Cards}

IBZ. - At least three cards are required by subroutine ZEBRA. The first is a ZEBRA card, in the notation of Table 3 . It defines a set of origins, beginning with the origin of subscript $J l$ and ending with $M X J$. The second or FIELD card, with $I B Z=0$ or 1 , prescribes a net of points to be set up about each origin in turn, at which certain field functions are to be computed. The last two cards must be of this type, since the calculation of a field net always returns to (3) for a new FIELD card. For exit to M.P., set $I B Z=-2$, on an otherwise blank card. To compute a second or third field net from the same origins, set again $I B Z=0$ or 1 . To change to a fresh set of origins, call a new ZEBRA card by setting IBZ $=-1$.

Apart from the switching function, non-negative entries in IBZ and IBR specify the field functions that arc to be computed. All functions called for, whether by IBZ or IBR, are computed before returning to (3) for a new FIELD card. The $z$ - and $\rho$ - intervals, and the range of coordinates are piescriber by the last six entries on the card (see Table 3). Dimensions are In inches if $R X=2.54$, in $\mathrm{cm}$ if $\mathrm{RX}$ is blank. The field calculations are further restricted by RHM, which limits the ratio, in percent, of the polar radius of the field point to that of the source nearest the origin.

The field series are truncated at order MAXN, or earlier if the sum of absolute magnitudes of the last 6 terms computed, when expressed in parts per million, is less than EPS. This monitoring sum is printed in either case. If it should be desired to study the details of convergence, either initial or final series terms may be printed by making an entry in IS, as specified in Table 3. The liigh-order series will not print in any case unless the ratio of polar radii exceeds RHP.
THE PROBLEM SETUP. FIRST PROBLEM

Two typical problems of different types will be considered. The first requires a calculation of the axial and radial components of the magnetic field, and the vector potential, at a number of points distributed throughout the interior of a system having a plane of symmetry, and consisting of five coaxial pairs of thick cylindrical coils. All fields are to be scaled to the value $B_{z}=1$. at $Z=0, \rho=0$. Suppose further that the system is comparatively long, with "mirror" coils of small diameter at the ends, and that it is desired to compute the field with high precision quite close to the windings. Hoving visualized the spheres of convergence, we may find it convenient to set up two ranges of equally spaced origins, for example from $z=0$ to $z=30$ at 6 -in. intervals, and from $z=33$ to $z=45$ at 3 -in。 intervals.

The main program requires a START card, five K-cards, and two ORIGN cards. On the START card, IX, J, BZI, and X may be blank. Also JO is blank on both ORIGN cards. That is, q-constants are wanted, the first origin is assigned to $J=1$, fields are normalized to $B_{z}=1$. of the origin $\mathrm{J}=1$, and no P-DUMP is desired. $K=5$, MAXN $=33, \mathrm{RX}=2.54$, since the coil dimensions were measured in inches. If the inner radii are of the order of 12 in., the choice RO $=10$. will lead to source constants that are conveniently printed by $F$-conversion. If dimensions were measured to .01 in., a scale factor of 100 will convert them all to integers, to ensure maximum precision. This factor, which is also applied below to Z0, AO, B, D, DLO, and O1, yields R.S $=1000$.

Since no derivatives are equired and all the coils are paired, the four integer fields are left blank on each $\mathrm{K}$-card. The current densities are in amp/in. ${ }^{2}$. On the first $O R I G N$ card, $B B=-1$, $\mathrm{DLO}=600$., $\mathrm{DLJ}=6$., while 01 and all the rest are blank. On the second card, DLO $=300$., DLJ $=5$, , O1 = 3300., and the rest are blank.

Sometimes only the source constants are required, without immediate field calculations. [See the sections on PUNNCH, READ, and TEST (M), below.] To bypass ZEBRA and return to START, set IB $=1$ on the last ORIGN card. (The PUNCH option may then be exercised, by setting $I X=-2$, not forgetting MAXN and RO.) If IB is blank, ZEBRA is called, but in either case 
source constants are first computed and printed for all origins.

If the field nets prescribed for the two ranges of origin are different, ZEBRA needs six cards to complete the problem, otherwise three suffice. To prescribe two types of field net, the first ZEBRA card may read $J I=1, M X J=6, M A X N=$ 33, $\mathrm{EPS}=1 ., \mathrm{RHM}=90 ., \mathrm{RX}=2.54$, with the rest blank. These options, with the highest possible order 33 , high limiting convergence ratio 0.90 , and error test of 1 part per million, as sume a need to compute the field with high precision and to include points quite close to the windings.

To calculate all three field functions but omit all extra print of the series terms, the first FIELD card should read $I B Z=1, I B R=1$, with $I S$ and DMY blank. The six numerical fields specify the desired ranges and intervals of the coordi. nates. These are true values, not scaled. Such a combination as $D L Z=2$., $Z M X=6 ., \mathrm{Zl}$ blank, computes fields at four points, with 2 -in. axial intervals, for each value of $\rho(R X=2.54)$. Every fourth calculation is redundant, since $z=6$ for one origin is the same as $z=0$ for the next. The field series, however, are quite distinct in the two cases, and at points very close to a field source, discrepancies arise from the truncation of the series, especially of the one for which the ratio RHO of polar rndii is lurger. Such checks are unnecessary, since in any case the print includes RHO, the terminal order $\mathrm{N}_{\text {, }}$ and the monitnring SUM of magnitudes of the last six lerms. It will be found, if a discrepaney oceurs, that nne series at least shows $N=M A X N$, and a SUM that is comparable to the observed error.

The program steps parallel to the axis through the whole range of $z$, including points referred to all origins, before $p$ is incremented. If a secimel field net is lestred lur the same origins, either to allow variable spaçing or to compute $\partial B \rho / \partial z$ in addition to $A$, add a second FIELD card. If not, an otherwise blank card that reads $I B Z=-1$ will call a new ZEBRA card. This may read the sume as before, except that for the second range of origins $J l=7, M X J=11$. This card is followed by one or more FIELD cards, then by an EXIT card with the single entry $I B Z=-2$, which returns to statement (1) of M.P. for a new START card. The PUNCH option may again be exercised at this time.

\section{SECOND PROBLEM}

The second problem is to compute the mutual inductance and axial force between one or more pairs of coils or of complete coaxial systems. Here the second stage of the problem is invariant, and it will be discussed first. It is carried out by TEST (M), using the field Eqs. (23) and (27) of Chapter 1, while ZEBRA is not called at all. TEST $(M)$ begins by computing $M J=M X J / 2$ and it assumes that subscripts $J=1,2, \ldots, M J$ identify the q-constants and minimum radii of a set of primary systems, while $M J+1, M J+2, \ldots$, MXJ identify the $p$-constants and maximum radii of the corresponding secondary systems. The stored data are thus matched by J subscript in paired sets, and a maximum of 12 mutual inductances and forces (each between a pair of elements or of complete systems) can be computed in one pass. If the ratio RHO of polar radii $\left(r_{\max }^{\prime} / r_{\min }\right)$ is less than unity, $F$ and $M$ are compuled, and are printed together with RHO. If not, $F$ and $M$ are set to zero. Optionally, the complete series may be printed; no series print, $F$ series only, or both, depending on whether $X=0 ., 1$. or 2 .

Mutual inductances are computed in henrys and forces in newtons. If the $C D$ entries on the data cards are current densities, the force may be read directly, but $M$ must be divided by the product of the currents. If they are turn densities, $F$ must be multiplied by the product of eurrents.

There is great flexibility in assembling data cards for M. P., to compute and store the constants in the first stage of these problems. The $J$-count must be iegulated to puir the systern subseripts properly. All START cards for primary systems, $J=1$ through $\frac{1}{2} M X J$, must have $I X=0$ for q-constants, while all the secondary cards have IX $=1$ for $p$-constants. The units used require $B Z 1=-1$ in both cases. Each systein muy have from 1 to 10 elminents, but with due rejurd to the convergence limitations on polai radii. ORIGN curds also must be carefully matched, since the field equations accept only $q$ 's and $p$ 's computed for the same origin.

\section{Case 1}

The mutual inductance and axial force are to be computed for a single pair of fixed coaxial coils, using a series of distinct origins. This severe test, after one or two trials have confirmed the validity of the method, will be reserved for critical cases in which the convergence ratio is very close to unity and a check on errors of 
truncation is desired. If two coils are close together, the convergence is best when the origin is near the point that leads to the smallest value of RHO. The optimum point can be located approximately by eye or by drawing arcs with a compass. When calculations are made from several origins, fairly far apart and lying on both sides of the chosen point, it is found that the optimum is quite flat.

The problem requires the standard set of three cards each for primary and secondary, plus one card to call TEST $(M)$. The two START cards differ only in IX and J. The overall range of magnitudes of the source constants, considering both $q_{n}$ and $p_{n}$, is least when RO is intermediate between the extreme radii of primary and secondary. $K=1, B Z 1=-1$, and if dimensions are in inches $R X=2.54$. For the primary, $I X$ and $J$ are blank, which calls for $q$-constants and assigns $J 1=1$. For the secondary, $\mid X=I, J=$ -1 . This continues the $J$-count with $J I=D L J+l$, where DLJ is the count on the ORIGN curd, but it also resets the cumulative maximum MXJ, which is necessary for proper functioning of TEST (M) if any earlier problem in the same deck has set $M X J$ too high. Otherwise, either $J=D L J$ or $J=-2$ would serve equally well. This question does not arise after MXJ has been once reset, but for the second START of a mutual inductance problem $\mathrm{J}=-1$ is the usual choice.

There is one K-card in each set. The two ORIGN cards are identical, with J0 blank, $B B=1$ to return to START, and the origins stepped as required. The last card calls TEST through IX $=-4$. It must repeat MAXN and $R X$, while $X=$ blank, 1.0 or 2.0 controls the optional series print.

\section{Case 2}

If instead of checking from several origins, it is desired to compute $M$ and $F$ for a pair of coils with variable axial spacing, the only change required is to enter separate values of DLO on the the two ORIGN cards, whose difference is the desired axial increment.

\section{Case 3}

As many as 12 independent pairs of elements or of complete systems or subsystems can be entered at one time, using up to 24 sets of START,
$K_{-}$, and ORIGN cards, before calling TEST (M). If a single primary or secondary subsystem is to be combined in turn with several others, the repeated system needs only one set of cards. The ORIGN card then carries in DLJ the number of combinations, while DLO is blank.

\section{THE FAP SECTION}

One essential subroutine is a FAP assembly of about 1000 words. All the Fortran sections can be effectively used, and even modified for special problems, without any detailed knowledge of this slave routine. It is called by FINDQK and ZEBRA, and by several versions of TEST that were written to tabulate the various zonal harmonic functions.

Except for some dozen words, the entire FAP section is written in fixed point, so as to retain 35 significant bits in the calculations, up to the point of return to the Fortran coding. It contains, in addition to short sections for conversions and tests, the series and recurrence routines for calculating the functions of polar angle, namely $P_{n}, P_{n}^{*}, U_{n}^{\star}, W_{n}^{*}$, and $\log [(1+s) / u]$. It also sums the contributions to $q_{1}$, namely $z_{L} \log [(1+s) / u]_{L}$, and to $q_{2}$, over the four sources of a rectangular coil ( $L=1$ to 4) with the full precision of the fixed point arithmetic, before floating the results for storage in COMMON.

\section{Normal Entry Points}

Special entry points for testing are not considered here. To calculate the functions of polar angle, the FAP section is entered at PNU, PNUM, or PUN, with suitoble data in each case to define the polar angle. In subroutine ZEBRA, which calculates various field components from the stored system constants, the normal 27-bit floating point precision suffices. The calling sequence is then:

$$
\begin{aligned}
& U=z / r \\
& S=\rho / \tau
\end{aligned}
$$

(Switch)

\section{CALL PNU}

The first two statements, with the coordinates on the right side replaced by their symbolic Fortran equivalents, compute the cosine and sine of the 
field-point polar angle. The "switch" is a function selector that specifies an output option (see below). Here the 27-bit floating point cosine $U$ defines the angular argument. To ensure the internal consistency of sine and cosine to 35 bits, the FAP section contains a one-step square root extraction on $1-U^{2}$, using the input value of $S$ as a trial divisor. The improved value is also re-floated and returned to $S$.

In critical coses, especially when calculating the source constants of long coil systems for a series of origins, it is desirable to use the full precision of the fixed point arithmetic. This requires that the polar angle be defined from the ratio of two coordinates that have been converted to exact floating point binary numbers from integral dimensions on the data cards, thus avoiding the error inherent in a decimal to binary fraction conversinn Tho syslem geometry is doccribed by a set of integers (exact binary fractions are also allowed) through the use of the arbitrary scale factor RS/RO, usually a power of 10 . The input lengths scaled $10 \mathrm{RS}$ are used solely to transmit exact ratios, through subroutine FINDQK, to the FAP section. The Fortran sections use thereafter lengths normalized on RO, which corresponds to the unit or reference length $r_{0}$ of Chapters 1 and 2.

Subroutine FINDQK transmits the scaled input eylindrical coordinates of each source to the FAP section, thus defining the angle through its tangent or cotongent:

$$
\begin{aligned}
& Z C=z \\
& A C=\rho
\end{aligned}
$$

(Switch)

\section{C.ALLL PNUM}

For precision testing, and for reserve flexibility, a third calling sequence is provided that is eui* rently used only in a polar version of subroutinc TEST:

$$
\begin{aligned}
& Z C=z \\
& A C=r \text { (polar) } \\
& \text { (Switch) } \\
& \text { CALL PUN }
\end{aligned}
$$

In either case the smaller of the scaled lengths $Z C$ and $A C$ is converted to a floating point mixed number (usually with one or more zero bits fol- lowing the binary point) having the same characteristic as the larger length. The latter retains its original form, in which the fractional part is always normalized. Both numbers are now converted to exact fixed point binary fractions by shifting left 8 bits to drop the characteristic. In effect, both are scaled down by the same unrecorded power of 2: This normalizing routine preserves only the ratio of lengths, while ensuring that the larger one contains 35 significant bits.

The calculation of sine and cosine is now easily completed in fixed point, followed by that of the remaining functions of polar angle. Evidently the PNUM sequence can generate valid 35-bit binary equivalents of cotangent or tangent arguments for such functions, and the PUN sequence similar equivalents of cosine arguments, when the arguimerits can be expreeced as rutlos of integers. In particular, 35-bit conversion of such decimal fractions as 0.57 or 0.6192 is correctly carried out.

\section{Output Options}

The functions that are transmitted to COMMON depend only on the switch instruction of the calling sequence and $n$ nt on whicli of the three sequences is used. In any case the FAP operations may be divided into two stages, the first of which generutes one of two series of fixed point functions und loads them into the internal array PX, while the second stage delivers one of five series of floating-point functions to the COMMON array QF. Fortran notation, with subscript $(N)$, will be used here for both arrays, while the order of the functions is shown by, the usual suthscript $n$.

Table 4 shows the effect of setting PN, SPNI, or WN to non-zero (NZ) as a switch instruction before the CALL statement. The switch must be reset for each entry, since all three locations are automatiratly zeranel before relurn. The functions that are storcd in PX and in QF are listed in the second and third columns, respectively.

In the first case, when the switch instruction is $P N=N Z$, the functions $P_{n}$ are first generated and stored in $\Gamma \times(\mathrm{N})$, beginning with $P_{1}=u$ in $P X$. The same functions are then floated and stored in $Q F(N+1)$. In all the other cases, the first step loads $-P_{n}^{\star}$ into $P X(N)$, beginning with $-s^{2}$ in PX, while the second step loads one of 
Table 4. Effect of Switch Options

\begin{tabular}{|c|c|c|c|c|}
\hline Switch & Fix & Float & $N(Q F)$ & $N(Q F)$ \\
\hline$(=N Z)$ & $P X(N)$ & $Q F(N)$ & Min & $\operatorname{Max}$ \\
\hline PN & $P_{n}$ & $P_{n-1}$ & 2 & $\operatorname{MAXN}+2$ \\
\hline SPNI (t) & $-P_{n}^{*}$ & $-P_{n-1}^{*}$ & 2 & $\operatorname{MAXN}+2$ \\
\hline SPNI (-) & $-P_{n}^{\star}$ & $-\frac{1}{n} P_{n-1}^{\prime}$ & 2 & $\operatorname{MAXN}+2$ \\
\hline WN & $-P_{n}^{*}$ & $W_{n-2}^{*}$ & 2 & $\operatorname{MAXN}+2$ \\
\hline None & $-P_{n}^{\star}$ & $U_{n}^{*}$ & 3 & MAXN \\
\hline
\end{tabular}

four different series into the array QF without disturbing the array PX. The integer MAXN, whose permissible values $5(4) 33$ have been previously noted, limits the range of all five recurrence routines.

Evidently if the calculation of $U_{n}^{\star}$ or $W_{n}^{*}$ or both, is to follow that of either set of the $P_{n}^{\prime}$ functions for the same argument, it would be redundant to repeat the iterations of the first step, that is, the calculation of $-P_{n}^{\star}$. For this reason a reentry instruction, CAL $n^{\circ}$ UM, is provided, which may or may not be preceded by the switch instruction $W M=N Z$. If the switch is set, the re-entry sequence computes $W_{n}^{*}$ if it is omitted, $U_{n}^{\star}$. Both options may be used if desired, in either order, and the redundant first step is bypassed each time.

\section{The Logarithmic Functions}

The logarithms required for $q_{1}$ and $q_{2}$, in the case of a coil of rectangular section, are computed with a precision of $3 \times 10^{-10}$ by a 4 term series approximation adapted from Hastings. Also, the fixed point consluints $q_{1}$ and $q_{2}$ are summed within the FAP section over the four sources of the coil. Only the sums are flouted, and stored in $Q F(1)$ and $Q F(2)$. The sequence of operations is controlled by the subscript $L$. This steps from 1 to 4 as the vertices of the coil cross-section are scanned by FINDQK in the order outer right $(+)$, inner right $(-)$, outer left $(-)$, inner left $(+)$. The L-sequence is diagramed in the box at the foot of Table 3.
Actually only two logarithms are computed, that of the ratio of $(1+s) / u$ for the sources 2 and 1 and aga in for 4 and 3 . To compute $q$, these logarithms must be multiplied by $z_{2}$ and $z_{1}$, respectively. This operation is carried out in fixed point after proper scaling of $z_{2}$ and $z_{1}$, using the same normalizing routine as was described above for ZC and AC. In this case, however, the scaling factor must be preserved, to be multiplied in floating point by the final sum after conversion. The logarithm routine computes onefourth of the true value in fixed point, and thus it is limited to arguments between 1 and $e^{-4}$. Therefore, the ratio of outer to inner cylindrical radius cannot exceed $e^{4}$ for any coil, or about 54.5. So high a ratio is unlikely to occur, but if it does an overflow signal is printed and the coil can be subdivided.

\section{Test Entry Points}

The entry points FTEST, LN, and LNT were provided for use with several versions of subroutine TEST, to permit critical testing of the FAP section beyond the limits of the 27 bit precision of floating point arithmetic. Details are omitted.

\section{OTHER SUBROUTINES}

ZEBRA (2) was written to supply vatues of $B_{z}$ and $B_{\rho}$ for single points whose cylindrical coordinates are placed in $Z C$ and $A C$, although it can also read in a complete field net, compute and print the field at each point. It uses the source constants stored in $Q S(J, N)$. It refers each field point automatically to the nearest origin, whother the origins are equally spaced or not, and it prints optionally.

PUNCH will punch out on data cards, six octal words to a card, either the complete set of origins with corresponding sets of source constants $Q S(J, N)$, or sets of field values $(B N)$ as described above under the IB field of the ORIGN card. There may be a recurring need for the source constants of a particular system, for exuimple, as iniput data for the calculation of particle orbits under various initial conditions. There is no need to recalculate the constants if they have been once punched out on cards, nor to reload the original data cards or any part of the zonal harmonic coding except subroutines READ and ZEBRA (2), for this purpose. The cards produced 
by PUNCH are loaded at the top of the data card deck.

Each of the two PUNCH options produces first an index card with a distinctive pattern for instant recognition. It is convenient to replace this card before use by a printed duplicate, as produced on a standard keypunch. In the case of the field data (BN), a separate index card is punched for each of the IB subsystems. This makes it easy to check the deck for completeness, after which all the index cards except the first must be removed. Each of the IB sets of field data is further divided by entries on the index card into $M 4$ groups of $M 3$ values each, representing field points at $M 3$ values of $z$ for each of $M 4$ values of $\rho$, as prescribed by the original FIELD card.

READ accepts sets of data cards produced by PUNCH and restores all variables to their original locations in COMMON. It also prints an indexed check list of the data, which duplicates the print of the original problem.

TEST was originally written in scveiul versions, tu test the precision of all parts of the program and to tabulate the several functions of polar angle, to cosine, cotangent, or tangent arguments. Details are omitted. TEST (M), which computes mutual inductances and axial forces between coaxial systems, was described in the section on problem setup.

OPEN is for general reserve. A completed version, offering a flexible PDUMP option, was discussed under heading $X$ of the main program.

BEST is currently a dummy subroutine, to be used in coding one or more field optimization programs. Two such routines were available before the present programs were written, but they will requirc revision of certain details, to accept the field data stored in $B N$ or recorded on eurds by PUNCH. Note that $B N$ has been placed at the end of COMMON storage, and that it is omitted from the list except in Subroutines ZEBRA, PUNCH, und READ. This makes it easy to alter its DIMENSION statement, if the present assignment $(8,100)$ proves inadequate. 


\section{BIBLIOGRAPHY}

(1) M. W. Garrett, J. Appl. Phys. 22, 1091-1107 (1951).

(2) M. W. Garrett, Tables of the Internal Magnetic Source Functions $U_{n}$ for Tbick Solenoids and Disk Coils, Edwards Brothers, Inc., Ann Arbor, 1953 (these tables are available upon request from the Thermonuclear Division of Oak Ridge National Laboratory).

(3) M. W. Garrett, Phys. Rev. 86, 613 (1952) (Abstract). A slightly different notation is used in this paper.

(4) N. B. Alexander and A. C. Downing, Tables for a Semi-Infinite Circular Current Sbeet, ORNL-2828 (1959).

(5) G. R. North and C. E. Parker, Thermonuclear Div. Progr. Rept. Oct. 31, 1961, ORNL-3239, p 77.

(G) J. C. Maxwell, Electricity and Magnetism, sec 697, Clarendon Press, Oxford, 1873.

(7) W. C. Elmore and M. W. Garrett, Rev. Sci. Instr. 25, 480-85 (1954).

(8) W. F. Gauster, Harold Mọtt, and C. E. Parker, Thermonuclear Project Semiann. Rept. July 31, 1960, ORNL-3011, p 85. 
THIS PAGE

WAS INTENTIONALLY

LEFT BLANK 
1. Biology Library

2-3. Central Research Library

4. Laboratory Shift Supervisor

5. Reactor Division Library

6-7. ORNL-Y-12 Technical Library

Document Reference Section

8-27. Laboratory Records Department

28. Laboratory Records, ORNL R.C.

29. P. S. Baker

30. W. A. Bell

31. R. E. Biggers

32-41. W. F. Gauster

42. R. F. Hibbs $(Y-12)$

43. C. E. Larson
44. J. A. Martin

45. J. P. Murray (K-25)

46-55. C. E. Parker

56. H. C. Schweinler

57. M. J. Skinner

58. A. H. Snell

59. J. A. Swartout

60. J. L. Wantland

61. A. M. Weinberg

62. W. P. Allis (consultant)

63-112. M. W. Garrett (consultant)

113. D. W. Kerst (consultant)

114. L. P. Smith (consultant)

\section{EXTERNAL DISTRIBUTION}

115. R. J. Mackin, Jr., Jet Propulsion Laboratory

116-185. AROD, Army Research Office, Durham, North Carolina

186. T. W. Sheheen, Aerojet General Nucleonics, P.O. Box 77, San Ramon, California

187. R. E. Ellis, Lawrence Radiation Laboratory, Livermore, California

188. E. P. Gray, Applied Physics Laboratory, Johns Hopkins University, 8621 Georgia Ave., Silver Spring, Maryland

189. Division of Research and Development, AEC, ORO

190-803. Given distribution as shown in TID-4500 (18th ed.) under Mathematics and Computers category (75 copies - OTS) 